\title{
Identification of an Elite Wheat-Rye T1RS-1BL Translocation Line Conferring High Resistance to Powdery Mildew and Stripe Rust
}

\author{
Guohao Han, ${ }^{1,5}$ Shiyu Liu, ${ }^{1,5}$ Jing Wang, ${ }^{1}$ Yuli Jin,,${ }^{1,5}$ Yilin Zhou, ${ }^{3}$ Qiaoling Luo, ${ }^{1,5}$ Hong Liu, ${ }^{1}$ He Zhao, ${ }^{2, \dagger}$ and \\ Diaoguo An $\mathbf{A n}^{\mathbf{1 , 4}, \dagger}$ \\ ${ }^{1}$ Center for Agricultural Resources Research, Institute of Genetics and Developmental Biology, Chinese Academy of Sciences, \\ Shijiazhuang 050021, Hebei, China \\ ${ }^{2}$ Institute of Genetics and Physiology, Hebei Academy of Agriculture and Forestry Science/Key Laboratory of Plant Genetic En- \\ gineering of Hebei Province, Shijiazhuang 050051, Hebei, China \\ ${ }^{3}$ The State Key Laboratory for Biology of Plant Disease and Insect Pests, Institute of Plant Protection, Chinese Academy of Ag- \\ ricultural Sciences, Beijing 100193, China \\ ${ }^{4}$ The Innovative Academy of Seed Design, Chinese Academy of Sciences, Beijing, China \\ ${ }^{5}$ University of Chinese Academy of Sciences, Beijing 100049, China
}

\begin{abstract}
Wheat-rye T1RS·1BL translocations have been widely used worldwide in wheat production for multiple disease resistance and superior yield traits. However, many T1RS·1BL translocations have successively lost their resistance to pathogens due to the coevolution of pathogen virulence with host resistance. Because of the extensive variation in rye (Secale cereale L.) as a naturally cross-pollinating relative of wheat, it still has promise to widen the variation of $1 \mathrm{RS}$ and to fully realize its application value in wheat improvement. In the present study, the wheat-rye breeding line R2207 was characterized by comprehensive

stable wheat-rye T1RS $1 \mathrm{BL}$ translocation line. Based on the disease responses to different isolates of powdery mildew and genetic analysis, R2207 appears to possess a novel variation for resistance, which was confirmed to be located on the rye chromosome arm 1RS. Line R2207 also exhibited high levels of resistance to stripe rust at both seedling and adult stages, as well as enhanced agronomic performance, so it has been transferred into a large number of commercial cultivars using an efficient 1RS-specific kompetitive allele specific PCR marker for markerassisted selection.
\end{abstract} analyses using genomic in situ hybridization (GISH), multicolor fluorescence in situ hybridization with multiple probes, multicolor GISH, and molecular marker analysis, and then was proven to be a cytogenetically
Keywords: powdery mildew, stripe rust, Secale cereale, Triticum aestivum, T1RS·1BL translocation, MAS, KASP
Powdery mildew and stripe rust, caused by Blumeria graminis (DC.) E.O. f. sp. tritici (Bgt) and Puccinia striiformis Westend. f. sp. tritici (Pst), respectively, are two devastating diseases of wheat (Triticum aestivum L.), significantly reducing grain yield and quality worldwide (Chen 2014; Dean et al. 2012). Host resistance is considered to be the most efficient, cost-effective, and environmentally friendly measure to control these diseases (Chen 2013; Ma et al. 2020). Up to now, more than 80 formally designated powdery mildew $(\mathrm{Pm})$ resistance genes and alleles $(\mathrm{Pml}$ to $\mathrm{Pm} 66)$ and more than 80 formally designated stripe rust $(\mathrm{Yr})$ resistance genes $(\mathrm{Yr} 1$ to $\mathrm{Yr} 83$ ) have been identified, but few of them have actually been applied in large-scale commercial production (Li et al. 2020a, 2020b; McIntosh et al. 2019; Qie et al. 2019; Tan et al. 2018). Due to the coevolution of pathogen virulence with host resistance, many race-specific genes have been quickly overcome by the new virulent pathotypes, particularly in areas where resistance genes were widely used (An et al. 2019). For instance, $P m 3 a, P m 8$, and $P m 17$ have been reported to have lost their resistance in the southeastern soft red winter wheat region in the United States (Cowger et al. 2009, 2018; Parks et al. 2008); Yr10 and Yr26, which were effective against all previously

${ }^{\dagger}$ Corresponding authors: H. Zhao; hezhao311@163.com and D. G. An; dgan@sjziam.ac.cn

Funding: This research was supported by the Strategic Priority Research Program of the Chinese Academy of Sciences No. XDA24030102, the National Natural Science Foundation of China No. 31771793, and the National Key Research and Development Program of China No. 2016YFD0102002.

The author(s) declare no conflict of interest.

Accepted for publication 2 May 2020.

() 2020 The American Phytopathological Society identified Pst races, have been defeated by the new $P s t$ race CYR34/V26 (Qi et al. 2016). Therefore, exploring new gene resources for powdery mildew and stripe rust resistance is urgently needed for improvement of wheat.

For a long time, conventional interbreeding between wheat cultivars and large-scale use of a few limited resistance genes has greatly narrowed the genetic basis of wheat resistance, resulting in a bottleneck in wheat improvement (Gupta et al. 2010). Wheat relatives have significant genetic diversity and abundant valuable genes; therefore, they play an important role in wheat improvement (Mujeeb-Kazi et al. 2013). A successful example of the introgression of elite alien genes into common wheat was the utilization of chromosome arm $1 \mathrm{RS}$ of rye (Secale cereale L., $2 \mathrm{n}=2 \mathrm{x}=14$, RR) (Lukaszewski 1990; Tester and Langridge 2010). That translocation derived from the rye cultivar Petkus carried valuable resistance genes, including $\operatorname{Pm} 8$ against powdery mildew, $\operatorname{Yr} 9$ against stripe rust, $\operatorname{Lr} 26$ against leaf rust, and Sr31 against stem rust (Mago et al. 2005; McIntosh et al. 2011), as well as genes associated with superior agronomic traits and abiotic-stress tolerance (Howell et al. 2014, 2019; Kumlay et al. 2003; Shearman et al. 2005). For those reasons, the wheat-rye T1RS $1 \mathrm{BL}$ translocations have been used worldwide in breeding programs. Approximately 1,050 wheat cultivars that carry T1RS $\cdot 1 \mathrm{BL}$ translocation inherit the chromosome arm 1RS of the rye cultivar Petkus (Schneider et al. 2016), which indicates that few resources in the rye species have served as the $1 \mathrm{RS}$ ancestors in current wheat cultivars (Kim et al. 2004). Owing to the narrow genetic basis and their long-term use in production, the resistance genes $\operatorname{Pm} 8, \operatorname{Yr} 9$, and Lr26 have successively lost their resistance to new virulent pathotypes of diseases since the 1990s (Lutz et al. 1992), and later virulence for $\operatorname{Sr} 31$ was reported (Pretorius et al. 2000, 2010). Despite the breakdown of resistance, the T1RS $1 \mathrm{BL}$ translocations have continued to be widely utilized in wheat breeding because of their yield potential and wide environmental adaptability (Ren et al. 2017; Wang et al. 2017). In addition, rye is a cross-pollinating crop, so it 
contains significant genetic heterogeneity within and among cultivars (Targońska et al. 2016). Abundance of genetic variations in $1 \mathrm{RS}$, such as $S r 50$ from the rye cultivar Imperial and $S r 1 R S^{A m i g o}$ from the rye cultivar Insave, still provide high resistance to the highly virulent wheat stem rust race $\mathrm{Ug} 99$, whereas the highly resistant gene Sr31 has been overcome by Ug99 (Mago et al. 2015; Rahmatov et al. 2016). Therefore, it is still important to unceasingly widen the variation of $1 R S$ to achieve the full potential of $1 R S$ in wheat improvement.

Once the alien chromatin was integrated into the wheat genome, genomic in situ hybridization (GISH) and fluorescence in situ hybridization (FISH) were widely used as effective detection methods to identify and analyze the chromosome composition due to their ease of use and accuracy (An et al. 2006, 2013). Meanwhile, molecular markers specific for alien chromosomes also have been powerful for detecting alien chromatin in a wheat background (Hechanova et al. 2018).

Combined with GISH/FISH, efficient alien-chromosome-specific markers have been extensively utilized to accelerate the process and improve the outcome of wheat-alien breeding programs (Edet et al. 2018). With the increased alien segments introduced into common wheat, it is critical to use tightly linked, high-resolution, and stable alien-specific markers to rapidly and efficiently detect the targeted progenies, especially the kompetitive allele specific PCR (KASP) markers, which have been widely used in gene mapping, and marker-assisted selection (MAS), due to their advantages of high throughput, high flexibility, desirable genetic stability, and locus specificity (Rasheed et al. 2016; Semagn et al. 2014; Wu et al. 2017).

A wheat-rye breeding line, R2207, exhibited high resistance to diseases and elite agronomic traits in wheat-growing regions for consecutive years. The objectives of this study were to (i) analyze the chromosome composition of R2207 using molecular cytogenetic methods; (ii) assess its resistance to powdery mildew and stripe rust isolates and analyze the inheritance of its resistance gene(s); (iii) survey the agronomic traits for breeding availability evaluation; and (iv) transfer R2207 into a large number of commercial cultivars using a 1RS-specific KASP marker for MAS breeding.

\section{Materials and Methods}

Plant materials. Line R2207 is a wheat-rye progeny of unknown pedigree. For consecutive years, it showed high resistance to powdery mildew and stripe rust in the field. Wheat cultivar Mingxian169, which does not have any documented $\mathrm{Pm}$ or $\mathrm{Yr}$ genes, was used as the susceptible control in the disease resistance tests for powdery mildew and stripe rust. Thirty-eight wheat genotypes carrying documented $P m$ gene(s) were used as controls for comparison with the powdery mildew resistance of R2207. The susceptible wheat cultivar Shixin 733 was crossed with $\mathrm{R} 2207$ to generate $\mathrm{F}_{2}$ progenies to determine the inheritance of the resistance of powdery mildew.

Total genomic DNA of Chinese Spring (CS) was used as a blocking DNA in GISH detection. Total genomic DNA of the rye cultivar Imperial, Triticum urartu $(2 \mathrm{n}=2 \mathrm{x}=14$, AA), Aegilops speltoides $(2 \mathrm{n}=2 \mathrm{x}=14, \mathrm{BB})$, and $A$. tauschii $(2 \mathrm{n}=2 \mathrm{x}=14$, DD) were used in multicolor GISH (mc-GISH) detection. For molecular marker analysis of R2207, CS, rye cultivar Imperial, the complete set of disomic addition lines of CS-Imperial, 1RS and 1RL ditelosomic addition lines of CS-Imperial, two triticale lines (10R2-193-2 and 10R2-194-2 [AABBRR]), and two T1BL·1RS wheat-rye translocation lines (Lovrin10 and Lovrin13) (Rabinovich 1998) were used as controls. All the seedlings were used for extracting total genomic DNA by the CTAB method (Gill et al. 1991).

Three wheat cultivars with the T1RS $1 \mathrm{BL}$ translocation, including Shimai18, Ji5265, and Heng4444, and three wheat cultivars without the T1RS·1BL translocation, including Shixin633, Shixin733, and Shixin828, were compared with R2207 to evaluate its agronomic traits in the field from 2015 to 2017.

To transfer the valuable genes into commercial cultivars, R2207 was crossed with 12 cultivars including Shixin633, Shixin733, Shixin828, Gao8901, Shiyou17, Shixin5071, Lumai13, Liangxing99, Jimai20, Jimai22, Tainong19, and Shannong17. CS and the rye cultivar Imperial were used as controls for genotyping of the 1RSspecific KASP markers.

Sequential GISH and multicolor FISH (mc-FISH) analyses. GISH and mc-FISH analyses were conducted to determine the chromosomal composition of the wheat-rye line R2207. For GISH analysis, the root tip cells were prepared for mitotic chromosome observation and hybridization as previously described (Han et al. 2009). Total genomic DNA of the rye cultivar Imperial was labeled with fluorescein-12-dUTP (green) by the nick translation method and used as a probe, whereas genomic DNA of CS was used as block with a 1:50 ratio of probe to blocker.

After GISH analysis, two highly repetitive sequence clones, pAs1 (red) labeled with digoxigenin-11-dUTP and pSc119.2 (green) labeled with biotin-11-dUTP, respectively, were used as probes for mc-FISH (Zheng et al. 2014). Nondenaturing FISH (ND-FISH) analysis also was performed to determine the identity of the rye and wheat chromosomes after GISH analysis. The combination of the oligonucleotide probes Oligo-pSc119.2-2 and Oligo-pTa535-2 could distinguish all the 42 chromosomes of wheat (Tang et al. 2014). The probe Oligo-pSc119.2-2 (green) was 5' end-labeled with 6-carboxyfluorescein, and the probe Oligo-pTa535-2 (red) was $5^{\prime}$ end-labeled with 6-carboxytetramethylrhodamine. These oligonucleotide probes were synthesized by Shanghai Invitrogen Biotechnology Co. (Shanghai, China). ND-FISH analysis was carried out according to the method of Tang et al. (2014).

All slides were counterstained with 4,6-diamidino-2-phenylindole after GISH or FISH. Then, detection and visualization of rye chromatin through an epifluorescence Olympus BX53 with a cooled chargecoupled device digital camera were performed. Program CellSens Standard 1.12 (Olympus Corporation, Tokyo, Japan) was used in image analysis.

mc-GISH analysis. Total genomic DNA of the rye cultivar Imperial and T. urartu labeled with fluorescein-12-dUTP, total genomic DNA of A. tauschii labeled with Texas-red-5-dUTP, and total genomic DNA of $A$. speltoides conducted as blocking (Fu et al. 2012) were used in combination for mc-GISH to determine the genomic identity of R2207. Image capture and analysis were performed as described above.

Molecular marker analysis. The $1 \mathrm{RS}$-specific marker O-SEC5'A/O-SEC3'-R (F, CTATTAGTTCGAAAAGCTTATGA; R, GCA TATGACTCAAATTATTTTTT) was used to detect 1RS chromatin (Shimizu et al. 1997). The marker O11B3/O11B5 (F, GGTACC AACAACAACAACCC; R, GTTGCTGCTGAGGTTGGTTC) was used as a diagnostic marker to detect the 1BS chromosome arm for the Glu-B3 gene (Van Campenhout et al. 1995). In molecular marker analysis, three offspring plants of R2207 from three consecutive generations were involved to confirm its genetic stability. CS, rye cultivar Imperial, the complete set of disomic addition lines of CS-Imperial, and 1RS and 1RL ditelosomic addition lines of CS-Imperial were used as controls to ensure the specificity of the 1RS-specific marker OSEC5'-A/O-SEC3'-R. Lovrin10 and Lovrin13 derived from rye cultivar Petkus (Rabinovich 1998) were used as controls because they are well-known T1RS·1BL translocation lines. Two triticale lines (10R2193-2 and 10R2-194-2) of unknown origin of rye were also used as controls to indicate the universality of the marker O-SEC5'-A/O$\mathrm{SEC} 3^{\prime}-\mathrm{R}$ in different rye resources.

The PCR amplification system $(10 \mu l$ total $)$ contains $1 \mu l$ of template DNA (50 to $150 \mathrm{ng} / \mu \mathrm{l}$ ), $4 \mu \mathrm{l}$ of $2 \times$ Taq Master Mix (Vazyme Biological, Nanjing, China), $4 \mu \mathrm{l}$ of $\mathrm{ddH}_{2} \mathrm{O}$, and $1 \mu \mathrm{l}$ of primers (10 $\mu \mathrm{mol})$. The PCR procedure was as follows: $94^{\circ} \mathrm{C}$ for $5 \mathrm{~min}$, followed by 36 cycles of $94^{\circ} \mathrm{C}$ for $30 \mathrm{~s}, 51^{\circ} \mathrm{C}\left(\mathrm{O}-\mathrm{SEC} 5^{\prime}-\mathrm{A} / \mathrm{O}-\mathrm{SEC} 3^{\prime}-\mathrm{R}\right)$ or $60^{\circ} \mathrm{C}(\mathrm{O} 11 \mathrm{~B} 3 / \mathrm{O} 11 \mathrm{~B} 5)$ for $30 \mathrm{~s}$, and $72^{\circ} \mathrm{C}$ for $40 \mathrm{~s}$, and a final extension at $72^{\circ} \mathrm{C}$ for $10 \mathrm{~min}$. The PCR products were separated in $2 \%$ agarose gel in $1 \times$ TAE buffer.

Assessment of resistance to powdery mildew and stripe rust. Line R2207 was tested for seedling resistance to powdery mildew with 23 single-pustule-derived $B g t$ isolates by separate inoculations in a greenhouse, along with 38 wheat genotypes carrying known Pm gene(s) as controls (Table 1). Twenty seeds of each line were planted in rectangular trays $(54 \times 28 \times 4 \mathrm{~cm})$ with 128 wells 
$(3 \times 3 \times 4 \mathrm{~cm})$, which were placed in the incubator, which was set at $20 \pm 0.5^{\circ} \mathrm{C}$ with a daily photoperiod of $14 \mathrm{~h}$. The susceptible control Mingxian 169 also was randomly planted in each tray. At the one-leaf stage, all seedlings were separately inoculated with fresh spores of single Bgt isolates that were increased on Mingxian169 seedlings and were incubated in darkness at $18 \pm 0.5^{\circ} \mathrm{C}$ with a relative humidity $>80 \%$ for $24 \mathrm{~h}$. The trays were subsequently transferred into a greenhouse with a diurnal cycle at $22 \pm 2{ }^{\circ} \mathrm{C}$ for $14 \mathrm{~h}$ of light and $18 \pm 2{ }^{\circ} \mathrm{C}$ for $10 \mathrm{~h}$ of darkness (Qie et al. 2019). When spore formation was observed on the first leaf of Mingxian169, the infection types (ITs) of powdery mildew at the seedling stage were scored using a 0 to 4 scale as described by $\mathrm{Si}$ et al. (1992), where 0 to 2 were considered resistant and 3 to 4 susceptible. To assess the resistance at the adult stage, R2207 and Mingxian169 were planted and inoculated with mixed Bgt isolates collected from northern China from 2014 to 2017 using three replicates at Luancheng Agro-Ecological Experimental Station, Chinese Academy of Sciences, Shijiazhuang, China. For each replicate, line R2207 and Mingxian169 were planted in two rows with 20 seeds per row. After the wheat reached full heading stage (Zadoks et al. 1974), the powdery mildew reactions for all the plants of R2207 and Mingxian169 were scored using a 0 to 9 scale, where 0 to 4 were considered resistant and 5 to 9 susceptible (Sheng and Duan 1991). In addition, a prevalent Bgt isolate, E09 (Li et al. 2011), was selected to test the reactions of seedlings of R2207, Shixin733, and their $F_{2}$ plants for the genetic analysis of the resistance. After evaluating the seedling reactions, these plants were transplanted into the field to test their adult-plant reactions, respectively.

Four Pst races (CYR31, CYR32, CYR33, and CYR34), which are prevalent and considered as highly pathogenic in China (Chen et al. 2009, 2014; Gebreslasie et al. 2020; Han et al. 2015; Wan et al. 2004), were used to assess the response of R2207 to stripe rust at the seedling stage in another greenhouse. The environmental conditions and methods of disease inoculation were previously described in Xu et al. (2014). Wheat cultivar Mingxian169 was used as a susceptible control. The IT was recorded based on a 0 to 4 scale in which 0 to 2 were considered resistant and 3 to 4 susceptible (Volin and Sharp 1973). At the adult stage, a mixture of the Pst races CYR31, CYR32, and CYR33 was used for artificial inoculation from 2014 to 2017 for R2207 and susceptible control Mingxian169 (Wang et al. 2009) at Luancheng Agro-Ecological Experimental Station, Chinese Academy of Sciences, Shijiazhuang, China. The field trials were conducted the same as that for assessment of adult-plant resistance to powdery mildew. The IT of stripe rust at the adult stage was scored based on the 0 to 9 scale as described by Wan et al. (2004).

Evaluation of agronomic traits. The wheat-rye line R2207 and six winter wheat cultivars including Shimai18, Ji5265, Heng4444, Shixin633, Shixin733, and Shixin828 were planted at the Luancheng Agro-Ecological Experimental Station, Chinese Academy of Sciences, Shijiazhuang, China. Shimai18, Ji5265, and Heng4444 contain T1RS·1BL translocations, whereas Shixin633, Shixin733, and Shixin828 do not. An evaluation of agronomic traits was conducted

Table 1. Seedling disease responses of R2207, susceptible wheat cultivar Mingxian169, and 38 wheat genotypes possessing documented powdery mildew resistance (Pm) genes to 23 individual Blumeria graminis f. sp. tritici $($ Bgt $)$ isolates ${ }^{\mathrm{z}}$

\begin{tabular}{|c|c|c|c|c|c|c|c|c|c|c|c|c|c|c|c|c|c|c|c|c|c|c|c|c|c|}
\hline \multirow[b]{2}{*}{ Cultivars/lines } & \multirow[b]{2}{*}{$P m$ genes } & \multirow[b]{2}{*}{$\begin{array}{c}\mathrm{R} / \mathrm{S} \\
\text { ratio }\end{array}$} & \multicolumn{23}{|c|}{ Bgt isolates } \\
\hline & & & E01 & E02 & E05 & E06 & E07 & E09 & E11 & E13 & E15 & E16 & E17 & E18 & E20 & E21 & $\begin{array}{l}\text { E23- } \\
(1)\end{array}$ & $\begin{array}{c}\text { E23- } \\
\text { (2) }\end{array}$ & E26 & $\begin{array}{l}\text { E30- } \\
\text { (1) }\end{array}$ & $\begin{array}{l}\text { E30- } \\
(2)\end{array}$ & E31 & E32 & E49 & E50 \\
\hline R2207 & Unknown & $16 / 7$ & $\mathrm{R}$ & $\mathrm{S}$ & $\mathrm{R}$ & $\mathrm{S}$ & $\mathrm{R}$ & $\mathrm{R}$ & $\mathrm{R}$ & $S$ & $\mathrm{R}$ & $\mathrm{R}$ & $\mathrm{S}$ & $\mathrm{R}$ & $\mathrm{R}$ & $\mathrm{R}$ & $\mathrm{R}$ & $\mathrm{R}$ & $\mathrm{R}$ & $\mathrm{R}$ & $S$ & $\mathrm{~S}$ & $\mathrm{~S}$ & $\mathrm{R}$ & $\mathrm{R}$ \\
\hline Kavkaz & 8 & $5 / 18$ & $\mathrm{R}$ & $\mathrm{S}$ & $\mathrm{S}$ & $\mathrm{S}$ & S & $\mathrm{S}$ & $S$ & $\mathrm{~S}$ & $\mathrm{~S}$ & $\mathrm{~S}$ & $\mathrm{~S}$ & S & $S$ & $\mathrm{~S}$ & $\mathrm{R}$ & S & $S$ & $S$ & S & $\mathrm{S}$ & $\mathrm{R}$ & $\mathrm{R}$ & $\mathrm{R}$ \\
\hline Amigo & 17 & $1 / 22$ & $\mathrm{~S}$ & $\mathrm{~S}$ & $\mathrm{~S}$ & $\mathrm{R}$ & S & $\mathrm{S}$ & $\mathrm{S}$ & $S$ & $\mathrm{~S}$ & $\mathrm{~S}$ & $\mathrm{~S}$ & $\mathrm{~S}$ & $\mathrm{~S}$ & $\mathrm{~S}$ & $\mathrm{~S}$ & $\mathrm{~S}$ & $\mathrm{~S}$ & $\mathrm{~S}$ & S & $\mathrm{S}$ & $\mathrm{S}$ & $\mathrm{S}$ & $\mathrm{S}$ \\
\hline CI14189 & 7 & $0 / 23$ & S & $\mathrm{S}$ & $\mathrm{S}$ & $\mathrm{S}$ & S & $\mathrm{S}$ & $\mathrm{S}$ & $\mathrm{S}$ & $\mathrm{S}$ & $\mathrm{S}$ & $\mathrm{S}$ & $S$ & $S$ & $\mathrm{~S}$ & $\mathrm{~S}$ & $\mathrm{~S}$ & $S$ & $\mathrm{~S}$ & S & $\mathrm{S}$ & $\mathrm{S}$ & $\mathrm{S}$ & $\mathrm{S}$ \\
\hline $\begin{array}{l}\text { TAM104/ } \\
\text { Thatcher }\end{array}$ & 20 & $13 / 10$ & $S$ & $\mathrm{R}$ & $\mathrm{R}$ & $\mathrm{R}$ & $\mathrm{S}$ & $S$ & $\mathrm{R}$ & $\mathrm{R}$ & $\mathrm{R}$ & $\mathrm{S}$ & $\mathrm{R}$ & $\mathrm{S}$ & $\mathrm{R}$ & $\mathrm{S}$ & $\mathrm{S}$ & $\mathrm{R}$ & $\mathrm{R}$ & $\mathrm{R}$ & $\mathrm{R}$ & $\mathrm{S}$ & $\mathrm{S}$ & $\mathrm{S}$ & $\mathrm{R}$ \\
\hline Mingxian 169 & - & $0 / 23$ & $\mathrm{~S}$ & $\mathrm{~S}$ & $\mathrm{~S}$ & $\mathrm{~S}$ & $\mathrm{~S}$ & $\mathrm{~S}$ & $\mathrm{~S}$ & $\mathrm{~S}$ & $\mathrm{~S}$ & $\mathrm{~S}$ & $\mathrm{~S}$ & $\mathrm{~S}$ & $\mathrm{~S}$ & $\mathrm{~S}$ & $\mathrm{~S}$ & $\mathrm{~S}$ & S & $\mathrm{S}$ & $\mathrm{S}$ & $\mathrm{S}$ & $\mathrm{S}$ & $\mathrm{S}$ & $\mathrm{S}$ \\
\hline Funo & - & $0 / 23$ & $\mathrm{~S}$ & $\mathrm{~S}$ & $\mathrm{~S}$ & $\mathrm{~S}$ & $\mathrm{~S}$ & $\mathrm{~S}$ & $\mathrm{~S}$ & $\mathrm{~S}$ & $\mathrm{~S}$ & $\mathrm{~S}$ & $\mathrm{~S}$ & $\mathrm{~S}$ & $\mathrm{~S}$ & $\mathrm{~S}$ & $\mathrm{~S}$ & $\mathrm{~S}$ & $\mathrm{~S}$ & $\mathrm{~S}$ & $\mathrm{~S}$ & $\mathrm{~S}$ & $\mathrm{~S}$ & $\mathrm{~S}$ & $\mathrm{~S}$ \\
\hline Ulka/8cc & 2 & $19 / 4$ & $\mathrm{R}$ & $\mathrm{R}$ & $\mathrm{R}$ & $\mathrm{R}$ & $\mathrm{R}$ & $\mathrm{R}$ & $\mathrm{R}$ & $\mathrm{R}$ & $\mathrm{R}$ & $\mathrm{R}$ & $\mathrm{R}$ & $\mathrm{S}$ & $\mathrm{S}$ & $\mathrm{S}$ & $\mathrm{R}$ & $\mathrm{R}$ & $\mathrm{R}$ & $\mathrm{R}$ & $\mathrm{R}$ & $\mathrm{R}$ & $\mathrm{S}$ & $\mathrm{R}$ & $\mathrm{R}$ \\
\hline Maris Huntsman & $2+6$ & $19 / 4$ & $\mathrm{R}$ & $\mathrm{R}$ & $\mathrm{R}$ & $\mathrm{R}$ & $\mathrm{R}$ & $\mathrm{R}$ & $\mathrm{R}$ & $\mathrm{R}$ & $\mathrm{R}$ & $\mathrm{R}$ & $\mathrm{R}$ & $\mathrm{S}$ & $\mathrm{S}$ & $\mathrm{S}$ & $\mathrm{R}$ & $\mathrm{R}$ & $\mathrm{R}$ & $\mathrm{R}$ & $\mathrm{R}$ & $\mathrm{R}$ & $\mathrm{S}$ & $\mathrm{R}$ & $\mathrm{R}$ \\
\hline Baimianmai3 & $4+8$ & $18 / 5$ & $\mathrm{R}$ & $\mathrm{R}$ & $\mathrm{R}$ & $\mathrm{R}$ & $\mathrm{R}$ & $\mathrm{R}$ & $\mathrm{R}$ & $\mathrm{R}$ & $\mathrm{S}$ & $\mathrm{R}$ & $\mathrm{R}$ & $\mathrm{S}$ & $\mathrm{S}$ & $\mathrm{S}$ & $\mathrm{R}$ & $\mathrm{R}$ & $\mathrm{R}$ & $\mathrm{R}$ & $\mathrm{R}$ & $\mathrm{S}$ & $\mathrm{R}$ & $\mathrm{R}$ & $\mathrm{R}$ \\
\hline Xiaobaidongmai & $\mathrm{XBD}$ & $13 / 10$ & $\mathrm{R}$ & $\mathrm{S}$ & $\mathrm{R}$ & $\mathrm{S}$ & $\mathrm{S}$ & $\mathrm{R}$ & $\mathrm{S}$ & $S$ & $\mathrm{~S}$ & $\mathrm{R}$ & $\mathrm{S}$ & $\mathrm{R}$ & $\mathrm{R}$ & $\mathrm{S}$ & $\mathrm{S}$ & $\mathrm{R}$ & $\mathrm{R}$ & $\mathrm{R}$ & $\mathrm{R}$ & $\mathrm{R}$ & $\mathrm{S}$ & $\mathrm{R}$ & $\mathrm{R}$ \\
\hline Chancellor & - & $0 / 23$ & $\mathrm{~S}$ & S & $\mathrm{S}$ & $\mathrm{S}$ & $\mathrm{S}$ & $\mathrm{S}$ & $\mathrm{S}$ & $S$ & $\mathrm{~S}$ & $\mathrm{~S}$ & $\mathrm{~S}$ & $\mathrm{~S}$ & $\mathrm{~S}$ & $\mathrm{~S}$ & $\mathrm{~S}$ & $\mathrm{~S}$ & $\mathrm{~S}$ & $\mathrm{~S}$ & $\mathrm{~S}$ & $\mathrm{~S}$ & $\mathrm{~S}$ & $\mathrm{~S}$ & $\mathrm{~S}$ \\
\hline Axminster/8cc & $\mathrm{R}$ & $2 / 21$ & $\mathrm{~S}$ & $\mathrm{~S}$ & $\mathrm{~S}$ & $\mathrm{R}$ & $\mathrm{S}$ & $\mathrm{S}$ & $\mathrm{R}$ & S & $\mathrm{S}$ & $\mathrm{S}$ & $\mathrm{S}$ & $\mathrm{S}$ & $\mathrm{S}$ & $\mathrm{S}$ & $\mathrm{S}$ & $\mathrm{S}$ & S & $\mathrm{S}$ & $\mathrm{S}$ & $\mathrm{S}$ & $\mathrm{S}$ & $\mathrm{S}$ & $\mathrm{S}$ \\
\hline Asosan/8cc & $3 a$ & $2 / 21$ & $\mathrm{~S}$ & $S$ & $\mathrm{~S}$ & $\mathrm{~S}$ & $\mathrm{~S}$ & $S$ & $S$ & $S$ & $\mathrm{~S}$ & $\mathrm{~S}$ & $\mathrm{~S}$ & $\mathrm{~S}$ & $\mathrm{~S}$ & $\mathrm{~S}$ & $\mathrm{~S}$ & $S$ & S & $\mathrm{R}$ & $\mathrm{R}$ & $\mathrm{S}$ & $\mathrm{S}$ & $\mathrm{S}$ & $\mathrm{S}$ \\
\hline $\mathrm{Chul} / 8 \mathrm{cc}$ & $3 b$ & $5 / 18$ & $\mathrm{~S}$ & $\mathrm{R}$ & $\mathrm{S}$ & $\mathrm{R}$ & $\mathrm{R}$ & $S$ & $\mathrm{R}$ & $\mathrm{S}$ & $\mathrm{R}$ & $\mathrm{S}$ & $\mathrm{S}$ & $\mathrm{S}$ & $\mathrm{S}$ & $\mathrm{S}$ & $\mathrm{S}$ & $\mathrm{S}$ & $S$ & $\mathrm{~S}$ & $\mathrm{~S}$ & $\mathrm{~S}$ & $\mathrm{~S}$ & $\mathrm{~S}$ & $\mathrm{~S}$ \\
\hline Sonora/8cc & $3 \mathrm{c}$ & $1 / 22$ & $\mathrm{~S}$ & $\mathrm{~S}$ & $\mathrm{~S}$ & $\mathrm{~S}$ & $\mathrm{~S}$ & $S$ & $S$ & S & $\mathrm{R}$ & $\mathrm{S}$ & $S$ & $\mathrm{~S}$ & $\mathrm{~S}$ & $\mathrm{~S}$ & $\mathrm{~S}$ & $\mathrm{~S}$ & S & $\mathrm{S}$ & $\mathrm{S}$ & $\mathrm{S}$ & $\mathrm{S}$ & $\mathrm{S}$ & $\mathrm{S}$ \\
\hline Kolibri & $3 d$ & $8 / 15$ & $\mathrm{R}$ & $\mathrm{S}$ & $\mathrm{S}$ & $\mathrm{R}$ & $\mathrm{S}$ & $\mathrm{S}$ & $\mathrm{R}$ & $\mathrm{R}$ & $\mathrm{S}$ & $\mathrm{S}$ & $\mathrm{S}$ & $\mathrm{S}$ & $\mathrm{S}$ & $\mathrm{S}$ & $\mathrm{R}$ & $\mathrm{S}$ & S & $\mathrm{R}$ & $\mathrm{R}$ & $\mathrm{S}$ & $\mathrm{S}$ & $\mathrm{R}$ & $\mathrm{S}$ \\
\hline Mich.Amber/8cc & $3 f$ & $0 / 23$ & $\mathrm{~S}$ & $\mathrm{~S}$ & $\mathrm{~S}$ & $\mathrm{~S}$ & $\mathrm{~S}$ & $\mathrm{~S}$ & $\mathrm{~S}$ & $\mathrm{~S}$ & $\mathrm{~S}$ & $\mathrm{~S}$ & $\mathrm{~S}$ & $\mathrm{~S}$ & $\mathrm{~S}$ & $\mathrm{~S}$ & $\mathrm{~S}$ & $\mathrm{~S}$ & S & $\mathrm{S}$ & $\mathrm{S}$ & $\mathrm{S}$ & $\mathrm{S}$ & $\mathrm{S}$ & $\mathrm{S}$ \\
\hline W150 & $3 e$ & $0 / 23$ & $\mathrm{~S}$ & $\mathrm{~S}$ & $\mathrm{~S}$ & $\mathrm{~S}$ & $\mathrm{~S}$ & $S$ & $\mathrm{~S}$ & $S$ & $\mathrm{~S}$ & $\mathrm{~S}$ & $S$ & $\mathrm{~S}$ & $\mathrm{~S}$ & $\mathrm{~S}$ & $\mathrm{~S}$ & $\mathrm{~S}$ & S & $\mathrm{S}$ & S & $\mathrm{S}$ & S & $\mathrm{S}$ & $\mathrm{S}$ \\
\hline Khapli/8cc & $4 \mathrm{a}$ & $18 / 5$ & $\mathrm{R}$ & $\mathrm{R}$ & $\mathrm{R}$ & $\mathrm{R}$ & $\mathrm{R}$ & $\mathrm{R}$ & $\mathrm{R}$ & $\mathrm{R}$ & $\mathrm{S}$ & $\mathrm{R}$ & $\mathrm{R}$ & $\mathrm{S}$ & S & $\mathrm{S}$ & $\mathrm{R}$ & $\mathrm{R}$ & $\mathrm{R}$ & $\mathrm{R}$ & $\mathrm{R}$ & $\mathrm{S}$ & $\mathrm{R}$ & $\mathrm{R}$ & $\mathrm{R}$ \\
\hline Armada & $4 \mathrm{~b}$ & $19 / 4$ & $\mathrm{R}$ & $\mathrm{R}$ & $\mathrm{R}$ & $\mathrm{R}$ & $\mathrm{R}$ & $\mathrm{R}$ & $\mathrm{R}$ & $\mathrm{R}$ & $\mathrm{R}$ & $\mathrm{R}$ & $\mathrm{R}$ & $\mathrm{S}$ & $\mathrm{S}$ & $\mathrm{S}$ & $\mathrm{R}$ & $\mathrm{R}$ & $\mathrm{R}$ & $\mathrm{R}$ & $\mathrm{R}$ & $\mathrm{S}$ & $\mathrm{R}$ & $\mathrm{R}$ & $\mathrm{R}$ \\
\hline $81-7241$ & $4 \mathrm{c}$ & $18 / 5$ & $\mathrm{R}$ & $\mathrm{R}$ & $\mathrm{R}$ & $\mathrm{R}$ & $\mathrm{R}$ & $\mathrm{R}$ & $\mathrm{R}$ & $\mathrm{R}$ & $\mathrm{R}$ & $\mathrm{R}$ & $\mathrm{R}$ & $\mathrm{S}$ & $\mathrm{S}$ & $\mathrm{S}$ & $\mathrm{R}$ & $\mathrm{R}$ & $\mathrm{R}$ & $\mathrm{R}$ & $\mathrm{R}$ & $\mathrm{S}$ & $\mathrm{S}$ & $\mathrm{R}$ & $\mathrm{R}$ \\
\hline Hope/8cc & $5 \mathrm{a}$ & $0 / 23$ & $\mathrm{~S}$ & $\mathrm{~S}$ & $\mathrm{~S}$ & $\mathrm{~S}$ & $\mathrm{~S}$ & $\mathrm{~S}$ & $\mathrm{~S}$ & S & $\mathrm{S}$ & $\mathrm{S}$ & $\mathrm{S}$ & $\mathrm{S}$ & $\mathrm{S}$ & $\mathrm{S}$ & $\mathrm{S}$ & $\mathrm{S}$ & $\mathrm{S}$ & $\mathrm{S}$ & $\mathrm{S}$ & $\mathrm{S}$ & $\mathrm{S}$ & $\mathrm{S}$ & $\mathrm{S}$ \\
\hline Aquila & $5 b$ & $19 / 4$ & $\mathrm{R}$ & $\mathrm{R}$ & $\mathrm{R}$ & $\mathrm{R}$ & $\mathrm{R}$ & $\mathrm{R}$ & $\mathrm{R}$ & $\mathrm{R}$ & $\mathrm{R}$ & $\mathrm{R}$ & $\mathrm{R}$ & $\mathrm{S}$ & $S$ & $\mathrm{~S}$ & $\mathrm{R}$ & $\mathrm{R}$ & $\mathrm{R}$ & $\mathrm{R}$ & $\mathrm{R}$ & $\mathrm{R}$ & $\mathrm{S}$ & $\mathrm{R}$ & $\mathrm{R}$ \\
\hline Coker 983 & $5+6$ & $5 / 18$ & $\mathrm{~S}$ & $\mathrm{~S}$ & $\mathrm{R}$ & S & $S$ & $S$ & $\mathrm{~S}$ & $\mathrm{~S}$ & $\mathrm{R}$ & $\mathrm{S}$ & $\mathrm{S}$ & $\mathrm{S}$ & $S$ & $\mathrm{~S}$ & $\mathrm{~S}$ & $S$ & $\mathrm{R}$ & $\mathrm{R}$ & $\mathrm{R}$ & $\mathrm{S}$ & $\mathrm{S}$ & $\mathrm{S}$ & $\mathrm{S}$ \\
\hline Timgalen & 6 & $0 / 22$ & $\mathrm{~S}$ & $S$ & $\mathrm{~S}$ & - & $\mathrm{S}$ & $\mathrm{S}$ & $\mathrm{S}$ & $\mathrm{S}$ & $\mathrm{S}$ & $\mathrm{S}$ & $\mathrm{S}$ & $\mathrm{S}$ & $S$ & $\mathrm{~S}$ & $\mathrm{~S}$ & $\mathrm{~S}$ & $S$ & $\mathrm{~S}$ & $S$ & $\mathrm{~S}$ & $\mathrm{~S}$ & $\mathrm{~S}$ & $\mathrm{~S}$ \\
\hline Coker 747 & 6 & $0 / 23$ & $\mathrm{~S}$ & $\mathrm{~S}$ & $\mathrm{~S}$ & $S$ & $\mathrm{~S}$ & $S$ & $S$ & $S$ & $\mathrm{~S}$ & $\mathrm{~S}$ & $\mathrm{~S}$ & $S$ & $\mathrm{~S}$ & $\mathrm{~S}$ & $\mathrm{~S}$ & $\mathrm{~S}$ & $\mathrm{~S}$ & $\mathrm{~S}$ & $\mathrm{~S}$ & $\mathrm{~S}$ & $\mathrm{~S}$ & $\mathrm{~S}$ & $\mathrm{~S}$ \\
\hline Wembley & 12 & $23 / 0$ & $\mathrm{R}$ & $\mathrm{R}$ & $\mathrm{R}$ & $\mathrm{R}$ & $\mathrm{R}$ & $\mathrm{R}$ & $\mathrm{R}$ & $\mathrm{R}$ & $\mathrm{R}$ & $\mathrm{R}$ & $\mathrm{R}$ & $\mathrm{R}$ & $\mathrm{R}$ & $\mathrm{R}$ & $\mathrm{R}$ & $\mathrm{R}$ & $\mathrm{R}$ & $\mathrm{R}$ & $\mathrm{R}$ & $\mathrm{R}$ & $\mathrm{R}$ & $\mathrm{R}$ & $\mathrm{R}$ \\
\hline $\mathrm{R} 4 \mathrm{~A}$ & 13 & $15 / 7$ & $\mathrm{R}$ & $\mathrm{R}$ & $\mathrm{S}$ & $\mathrm{R}$ & $\mathrm{R}$ & $\mathrm{R}$ & $\mathrm{R}$ & $S$ & $\mathrm{~S}$ & $\mathrm{R}$ & $\mathrm{R}$ & - & $\mathrm{R}$ & $\mathrm{R}$ & $S$ & $\mathrm{R}$ & $\mathrm{R}$ & $S$ & $\mathrm{R}$ & $\mathrm{R}$ & $\mathrm{R}$ & $\mathrm{S}$ & $\mathrm{S}$ \\
\hline Brigand & 16 & $23 / 0$ & $\mathrm{R}$ & $\mathrm{R}$ & $\mathrm{R}$ & $\mathrm{R}$ & $\mathrm{R}$ & $\mathrm{R}$ & $\mathrm{R}$ & $\mathrm{R}$ & $\mathrm{R}$ & $\mathrm{R}$ & $\mathrm{R}$ & $\mathrm{R}$ & $\mathrm{R}$ & $\mathrm{R}$ & $\mathrm{R}$ & $\mathrm{R}$ & $\mathrm{R}$ & $\mathrm{R}$ & $\mathrm{R}$ & $\mathrm{R}$ & $\mathrm{R}$ & $\mathrm{R}$ & $\mathrm{R}$ \\
\hline XX186 & 19 & $0 / 23$ & $\mathrm{~S}$ & $\mathrm{~S}$ & $\mathrm{~S}$ & $S$ & $\mathrm{~S}$ & $\mathrm{~S}$ & $\mathrm{~S}$ & $\mathrm{~S}$ & $\mathrm{~S}$ & $\mathrm{~S}$ & $\mathrm{~S}$ & $\mathrm{~S}$ & $\mathrm{~S}$ & $\mathrm{~S}$ & $\mathrm{~S}$ & $\mathrm{~S}$ & $\mathrm{~S}$ & $\mathrm{~S}$ & $\mathrm{~S}$ & $\mathrm{~S}$ & $\mathrm{~S}$ & $\mathrm{~S}$ & $\mathrm{~S}$ \\
\hline $\operatorname{DS} 6 \mathrm{~V}(6 \mathrm{~A})$ & 21 & $22 / 0$ & $\mathrm{R}$ & $\mathrm{R}$ & $\mathrm{R}$ & $\mathrm{R}$ & $\mathrm{R}$ & $\mathrm{R}$ & $\mathrm{R}$ & $\mathrm{R}$ & $\mathrm{R}$ & $\mathrm{R}$ & $\mathrm{R}$ & $\mathrm{R}$ & $\mathrm{R}$ & $\mathrm{R}$ & $\mathrm{R}$ & $\mathrm{R}$ & $\mathrm{R}$ & $\mathrm{R}$ & $\mathrm{R}$ & $\mathrm{R}$ & $\mathrm{R}$ & $\mathrm{R}$ & - \\
\hline Chiyacao & 24 & $10 / 13$ & $\mathrm{R}$ & $\mathrm{R}$ & $\mathrm{S}$ & $\mathrm{S}$ & $\mathrm{R}$ & $\mathrm{R}$ & $\mathrm{R}$ & $\mathrm{S}$ & $\mathrm{S}$ & $\mathrm{R}$ & $\mathrm{R}$ & $\mathrm{S}$ & $S$ & $\mathrm{~S}$ & $\mathrm{~S}$ & $\mathrm{R}$ & $\mathrm{R}$ & $S$ & $\mathrm{R}$ & $\mathrm{S}$ & $\mathrm{S}$ & $S$ & $\mathrm{~S}$ \\
\hline Maris Dove & $2+\mathrm{MLD}$ & $18 / 4$ & $\mathrm{R}$ & $\mathrm{R}$ & $\mathrm{R}$ & - & $\mathrm{R}$ & $\mathrm{R}$ & $\mathrm{R}$ & $\mathrm{R}$ & $\mathrm{R}$ & $\mathrm{R}$ & $\mathrm{R}$ & $\mathrm{S}$ & $S$ & $\mathrm{~S}$ & $\mathrm{R}$ & $\mathrm{R}$ & $\mathrm{R}$ & $\mathrm{R}$ & $\mathrm{R}$ & $\mathrm{R}$ & $\mathrm{S}$ & $\mathrm{R}$ & $\mathrm{R}$ \\
\hline Mission & $4 b+5 b$ & $19 / 4$ & $\mathrm{R}$ & $\mathrm{R}$ & $\mathrm{R}$ & $\mathrm{R}$ & $\mathrm{R}$ & $\mathrm{R}$ & $\mathrm{R}$ & $\mathrm{R}$ & $\mathrm{R}$ & $\mathrm{R}$ & $\mathrm{R}$ & $S$ & $S$ & $\mathrm{~S}$ & $\mathrm{R}$ & $\mathrm{R}$ & $\mathrm{R}$ & $\mathrm{R}$ & $\mathrm{R}$ & $S$ & $\mathrm{R}$ & $\mathrm{R}$ & $\mathrm{R}$ \\
\hline Normandie & $1+2+9$ & $1 / 22$ & $S$ & $\mathrm{~S}$ & $\mathrm{~S}$ & S & $S$ & $S$ & $\mathrm{~S}$ & $S$ & $\mathrm{~S}$ & $\mathrm{~S}$ & $\mathrm{~S}$ & $\mathrm{~S}$ & $S$ & $\mathrm{~S}$ & $\mathrm{~S}$ & $\mathrm{~S}$ & $S$ & $S$ & $\mathrm{R}$ & $\mathrm{S}$ & $\mathrm{S}$ & $\mathrm{S}$ & $\mathrm{S}$ \\
\hline $5 \mathrm{P} 27$ & 30 & $18 / 4$ & $\mathrm{R}$ & $\mathrm{R}$ & $\mathrm{R}$ & - & $\mathrm{R}$ & $\mathrm{R}$ & $\mathrm{R}$ & $\mathrm{R}$ & $\mathrm{R}$ & $\mathrm{R}$ & $\mathrm{R}$ & $S$ & $S$ & $\mathrm{~S}$ & $\mathrm{R}$ & $\mathrm{R}$ & $\mathrm{R}$ & $\mathrm{R}$ & $\mathrm{R}$ & $\mathrm{S}$ & $\mathrm{R}$ & $\mathrm{R}$ & $\mathrm{R}$ \\
\hline NCA5 & 25 & $7 / 16$ & $\mathrm{~S}$ & $S$ & $\mathrm{~S}$ & $\mathrm{R}$ & $\mathrm{S}$ & $\mathrm{S}$ & $\mathrm{R}$ & $\mathrm{S}$ & $\mathrm{R}$ & $\mathrm{S}$ & $\mathrm{S}$ & $\mathrm{S}$ & $\mathrm{S}$ & $\mathrm{S}$ & $\mathrm{S}$ & $S$ & $\mathrm{~S}$ & $\mathrm{R}$ & $\mathrm{R}$ & $\mathrm{S}$ & $\mathrm{S}$ & $\mathrm{R}$ & $\mathrm{R}$ \\
\hline NCD7 & 34 & $9 / 14$ & $\mathrm{~S}$ & $\mathrm{R}$ & $\mathrm{R}$ & S & $\mathrm{S}$ & $S$ & $\mathrm{R}$ & $S$ & $\mathrm{R}$ & $\mathrm{S}$ & $\mathrm{R}$ & $\mathrm{S}$ & $S$ & $S$ & $\mathrm{~S}$ & $S$ & $\mathrm{R}$ & $\mathrm{R}$ & $\mathrm{R}$ & $\mathrm{S}$ & $\mathrm{S}$ & $\mathrm{S}$ & $\mathrm{R}$ \\
\hline NCD3 & 35 & $16 / 7$ & $\mathrm{R}$ & $\mathrm{R}$ & $\mathrm{R}$ & $S$ & $\mathrm{~S}$ & $S$ & $S$ & $S$ & $\mathrm{R}$ & $\mathrm{R}$ & $\mathrm{R}$ & $\mathrm{R}$ & $S$ & $\mathrm{~S}$ & $\mathrm{R}$ & $\mathrm{R}$ & $\mathrm{R}$ & $\mathrm{R}$ & $\mathrm{R}$ & $\mathrm{R}$ & $\mathrm{R}$ & $\mathrm{R}$ & $\mathrm{R}$ \\
\hline
\end{tabular}

${ }^{\mathrm{z}}$ Infection types of powdery mildew at seedling stage were scored using a 0 to 4 scale as $\mathrm{Si}$ et al. (1992) described, in which 0 to 2 were considered resistant (R) and 3 to 4 susceptible (S). Dash (-) indicates no data. 
from 2015 to 2017 with three replicates in a randomized complete block design. For each replicate, 20 seeds were evenly sown in 1.5 -m-long rows spaced $0.25 \mathrm{~m}$ apart. At physiological maturity (Zadoks et al. 1974), plant height, spike number per plant, spike length, fertile spikelet number per spike, kernel number per spike, thousand-kernel weight, and grain yield of 10 randomly selected plants in the middle of the two internal rows were investigated. The ANOVA and least significant difference test were performed using SPSS 22.0 software (SPSS, Chicago, IL) to test the significance of differences between R2207 and the six wheat cultivars for each agronomic trait.

Use of R2207 in wheat breeding. To transfer the valuable resistance and yield-associated genes into commercial wheat, R2207 was crossed and back-crossed with 12 widely grown cultivars in Hebei and Shandong provinces including Shixin633, Shixin733, Shixin828, Gao8901, Shiyou17, Shixin5071, Lumai13, Liangxing99, Jimai20, Jimai22, Tainong19, and Shannong17. Combined with the phenotype evaluation in the field, the 1RS-specific KASP marker SWK5282 (forward primer 1 with rye allele, gaaggtgaccaagttcatgctGAGCTGAT TTCCATGTA; forward primer 2 with wheat allele, gaaggtcggagtcaacggattGAGCTGATTTCCATGTC; and reverse primer, TACCA AGTCCTGAACCA) (Han et al. 2020) was used to efficiently trace $1 R S$ chromosomes in the $\mathrm{F}_{2}$ or $\mathrm{BC}_{1} \mathrm{~F}_{1}$ populations for MAS. This KASP marker is developed based on a $1 \mathrm{RS}$-specific single nucleotide polymorphism (SNP) allele between the rye and wheat genome, and it has been validated that it can be applied to screen wheat-rye derived populations on large scale, unconstrained by different wheat and rye backgrounds (Han et al. 2020).

The KASP amplification system ( $8 \mu$ l total) consisted of $4.5 \mu l$ standard-carboxy-X-rhodamine KASP master mix, $0.13 \mu$ l of assay mix, $0.97 \mu \mathrm{l}$ of $\mathrm{ddH}_{2} \mathrm{O}$, and $2.4 \mu \mathrm{l}$ of template DNA $(50 \mathrm{ng} / \mu \mathrm{l})$. The reaction was performed in a Bio-Rad CFX real-time PCR system (Bio-Rad Laboratories, Hercules, CA). The PCR procedure was as follows: $94^{\circ} \mathrm{C}$ for $15 \mathrm{~min}, 94^{\circ} \mathrm{C}$ for $20 \mathrm{~s}$, followed by 10 touchdown cycles of 64 to $58^{\circ} \mathrm{C}$ (decreasing $0.6^{\circ} \mathrm{C}$ per cycle), 38 cycles of $94^{\circ} \mathrm{C}$ for $20 \mathrm{~s}$, and $58^{\circ} \mathrm{C}$ for $60 \mathrm{~s}$, and fluorescence was detected using BioRad CFX Manage 3.1 software.

\section{Results}

Sequential GISH and FISH analyses of R2207. Using genomic DNA of the rye cultivar Imperial as a probe, GISH analysis showed one pair of intact rye chromosome arms, which displayed a strong green hybridization signal and contained an obvious secondary constriction, were translocated onto one pair of wheat chromosomes in R2207 with a total number of 42 chromosomes in a cell (Fig. 1A and B). After that, GISH analyses using the probes pAs1 combined with pSc119.2, and Oligo-pSc119.2-2 combined with OligopTa535-2, determined the identity of the individual chromosomes of R2207. The probes pSc119.2 or Oligo-pSc119.2-2 produced strong green hybridization signals in the telomere regions and weak
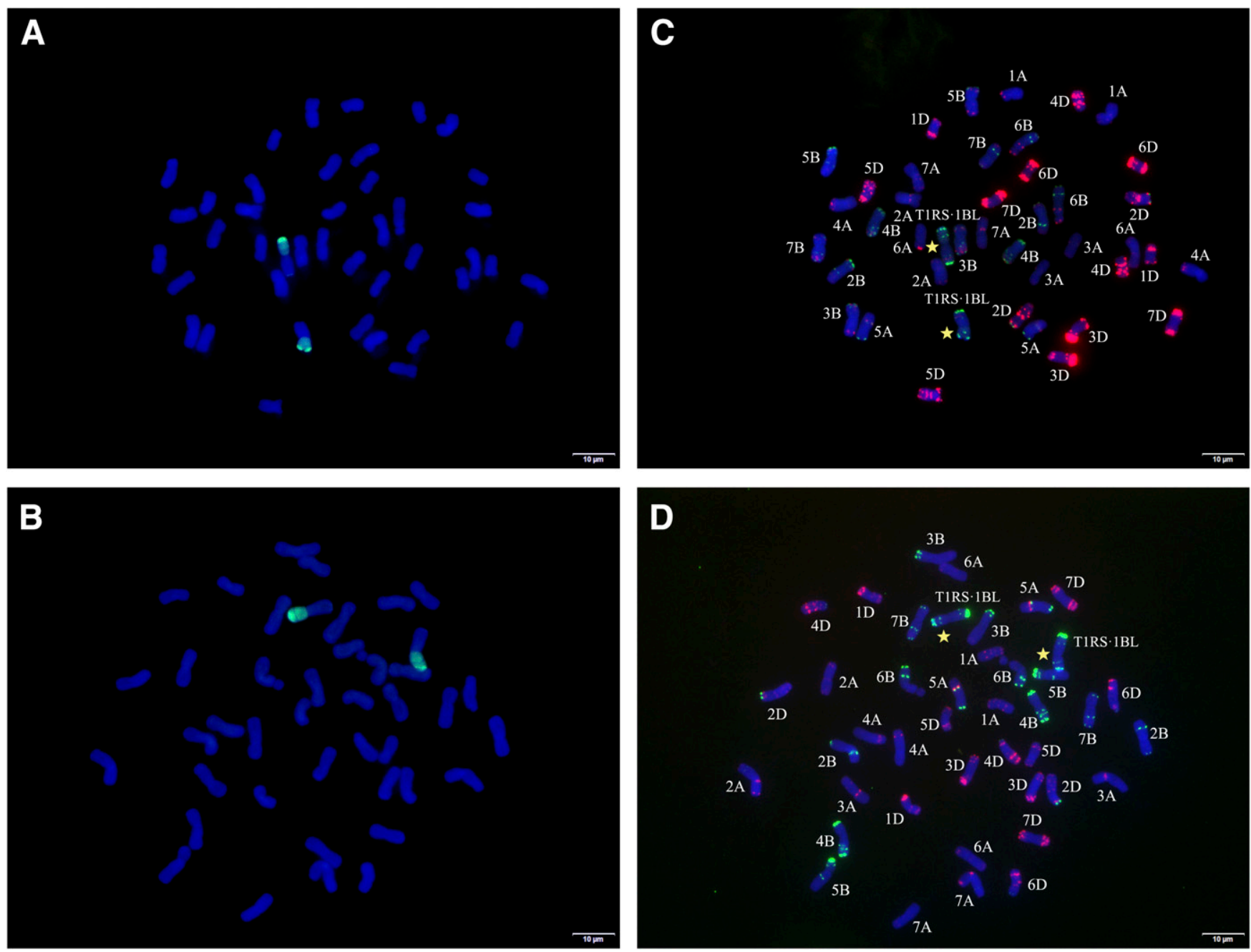

Fig. 1. Genomic in situ hybridization (GISH) and multicolor fluorescence in situ hybridization (mc-FISH) analyses of chromosomes of R2207. For GISH, total genomic DNA of the rye cultivar Imperial (green) were used as a probe and Chinese Spring DNA as a blocker. Chromosomes were counterstained with 4,6-diamidino-2-phenylindole (blue). A and B, GISH analysis of R2207. C, mc-FISH analysis of the same metaphase cell after GISH analysis (A) with pSc119.2 (green) and pAs1 (red). D, Nondenaturing FISH analysis of the same metaphase cell after GISH analysis (B) with Oligo-pSc119.2-2 (green) and Oligo-pTa535-2 (red). The bar represents $10 \mu \mathrm{m}$, and the stars indicate the translocation chromosomes T1RS·1BL. 
hybridization signals in the subtelomere regions on both the long and short arms of the translocated chromosomes, indicating that the rye chromosome arm 1RS had translocated onto the wheat chromosome arm 1BL, thereby forming a wheat-rye T1RS $1 \mathrm{BL}$ translocation line (Fig. 1C and D). The seeds from selfed R2207 were used for the sequential GISH and mc-FISH analyses in three consecutive generations. The results revealed that R2207 was a cytogenetically stable T1RS·1BL translocation line, and its chromosome composition was $2 \mathrm{n}=42=20^{\prime \prime} \mathrm{W}+1^{\prime \prime} \mathrm{T} 1 \mathrm{RS} \cdot 1 \mathrm{BL}$.

mc-GISH analysis of R2207. mc-GISH analysis was performed to further reveal the genomic identity of the chromosomes in R2207. Using genomic DNA of the rye cultivar Imperial and three diploid progenitors of common wheat as probes or blockers, the results of the cross-hybridization signal made the rye chromosomes display green fluorescent signals, whereas the wheat A-, B-, and D-genome chromosomes displayed yellow-green, brown or gray, and pink or red fluorescent signals, respectively. The results demonstrated that R2207 contained seven pairs of A-genome chromosomes (yellow-green), six pairs of B-genome chromosomes (brown or gray), and seven pairs of D-genome chromosomes (pink or red), along with a translocation of one pair of rye chromosome arms (green) to one pair of B-genome chromosomes (Fig. 2, arrows). Two chromosome genomic translocation events occurred in R2207. A standing chromosome arrangement event in hexaploid wheat that two small B-genome chromosome fragments translocated onto the two long arms of A-genome chromosomes (Dvorak et al. 2018) were detected (Fig. 2, diamonds). Simultaneously, two other small Bgenome chromosome fragments were translocated onto the two long arms of D-genome chromosomes (Fig. 2, stars).

Molecular marker analysis of R2207. Molecular marker analysis was also conducted to confirm the chromosome composition of R2207. The 1RS-specific marker O-SEC5'-A/O-SEC3'-R amplified two specific fragment bands consisting of approximately 1,530 and $700 \mathrm{bp}$. The 1BS-diagnostic marker O11B3/O11B5 amplified an approximate 630-bp fragment band. As expected, O-SEC5'-A/OSEC 3'-R amplified the same size of specific bands in the rye cultivar Imperial and three offspring plants of R2207 from three consecutive generations, $1 \mathrm{R}$ disomic addition line and $1 \mathrm{RS}$ ditelosomic addition line of CS-Imperial, two T1BL-1RS translocation lines (Lovrin10 and Lovrin13), and two triticale lines (10R2-193-2 and 10R2-194-2),

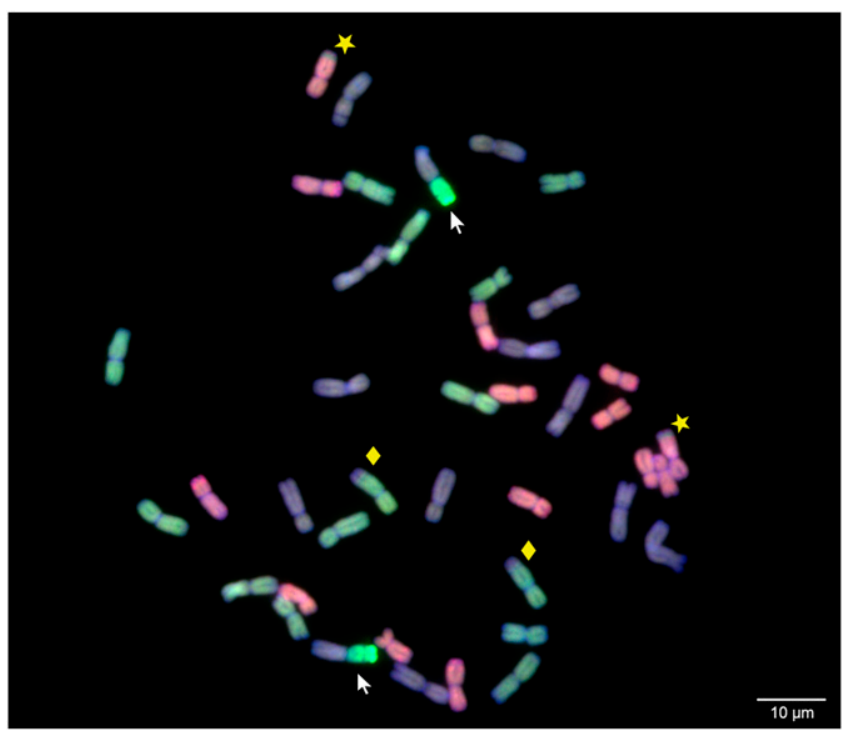

Fig. 2. Multicolor genomic in situ hybridization analysis of R2207. Bright-green, yellow-green, brown or gray, and pink or red fluorescence signals indicate R-genome, A-genome, B-genome, and D-genome chromosomes, respectively. Two small B-genome chromosome fragments are translocated to A-genome chromosomes (diamonds), and other two small B-genome chromosome fragments are translocated to D-genome (stars). The bar represents $10 \mu \mathrm{m}$, and the arrows indicate the translocation chromosomes T1RS·1BL. but not in CS or the wheat-rye disomic addition lines except for the $1 \mathrm{R}$ addition line or the 1RL ditelosomic addition line of CS-Imperial (Fig. 3A). Meanwhile, the marker O11B3/O11B5 did not amplify fragments in Imperial, three offspring plants of R2207 from three consecutive generations, or two T1BL·1RS translocation lines (Lovrin10 and Lovrin13), but amplification occurred in CS, a complete set of disomic addition lines and 1RS and 1RL ditelosomic addition lines of CSImperial, and two triticale lines (10R2-193-2 and 10R2-194-2) (Fig. 3B). These results demonstrated that the chromosome arm 1BS of R2207 had been substituted by the chromosome arm 1RS of rye.

Analyses of $\mathbf{R 2 2 0 7}$ for resistance to powdery mildew and stripe rust. At the seedling stage, line R2207 was highly resistant to 16 of 23 Bgt isolates, accounting for $69.6 \%$ of the isolates tested (Table 1). To confirm the source of resistance, the $F_{2}$ population of R2207 and susceptible cultivar Shixin 733 were used to test for powdery mildew resistance against isolate E09. The results demonstrated that all the plants containing $1 \mathrm{RS}$ showed the same highly resistant reaction as R2207, but the plants without 1RS were susceptible (Fig. 4). Thus, the powdery mildew resistance in R2207 was confirmed to have been derived from 1RS. Meanwhile, R2207 showed a significantly different reaction pattern in comparison with all the wheat genotypes. Especially, for the genotypes with documented $P m$ gene(s) derived from rye, Kavkaz with Pm8 derived from 1 RS of Petkus rye was resistant to five of 23 Bgt isolates, Amigo with Pm17 from 1RS of Insave rye was resistant to only one isolate, CI14189 with $P m 7$ from 2RL of Rosen rye was susceptible to all 23 isolates, and TAM104/Thatcher with Pm20 from 6RL of Prolific rye was resistant to 13 of $23 \mathrm{Bgt}$ isolates (Table 1), indicating that R2207 most likely carries a new resistance variation. At the adultplant stage, over three consecutive growing seasons, R2207 exhibited high resistance to a mixture of $B g t$ isolates in the field with an IT 0 to 2, whereas the control Mingxian 169 showed a highly susceptible reaction with an IT 8 to 9 . The corresponding $F_{2}$ plants of R2207 and Shixin733, which were transplanted to the field, showed a consistent disease response that agreed with that of the seedling resistance test.

Line R2207 showed high resistance to four stripe rust races (CYR31, CYR32, CYR33, and CYR34) at the seedling stage,

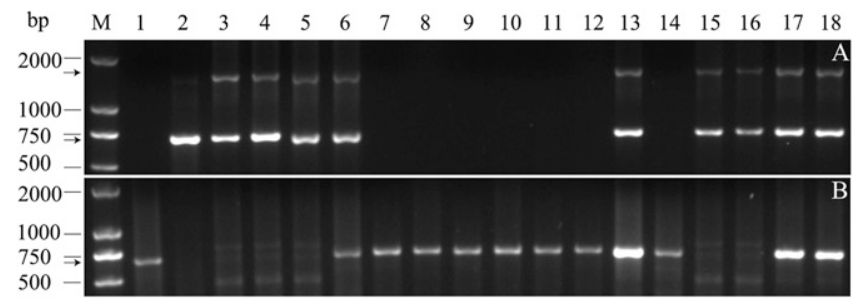

Fig. 3. $P C R$ amplification: A, 1RS-specific marker O-SEC5'-A/O-SEC3'-R; and B, 1BS-diagnostic marker 011B3/011B5. Lanes: $M=$ D2000; 1 = Chinese Spring (CS); $2=$ Imperial; 3 to $5=$ R2207 from three consecutive generations; 6 to $12=$ $1 \mathrm{R}$ to $7 \mathrm{R}$ disomic addition lines of CS-Imperial; 13 to $14=1 \mathrm{RS}$ and $1 \mathrm{RL}$ ditelosomic addition lines of CS-Imperial; 15 to $16=$ two T1BL-1RS translocation lines (Lovrin10 and Lovrin13); and 17 to $18=$ two triticale lines (10R2-193-2 and 10R2-194-2). The arrows indicate the diagnosed amplified fragments of the markers.

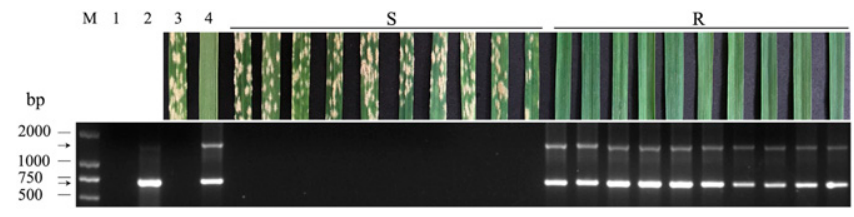

Fig. 4. Evaluation of powdery mildew resistance at seedling stage and molecular detection of the 1RS chromosome from R2207 using 1RS-specific markers OSEC5'-A/O-SEC3'-R in $F_{2}$ plants crossed by R2207 and Shixin733. M = D2000; $1=$ Chinese Spring; 2 = Imperial; $3=$ Shixin733; $4=$ R2207; and S and R = susceptible and resistant $F_{2}$ plants, respectively. The arrows indicate the diagnosed amplified fragment. 
whereas Mingxian169 was highly susceptible (Fig. 5, Table 2). Among the documented $\mathrm{Yr}$ genes, $\mathrm{Yr} 9$ that derived from $1 \mathrm{RS}$ of the rye cultivar Petkus was susceptible to the four races. Also, the widely virulent race CYR34 has overcome most of the $\mathrm{Yr}$ genes currently used in wheat production, such as $\operatorname{Yr} 26$ (Table 2). At the adultplant stage, R2207 also showed a high level of resistance to a mixture of $P s t$ races CYR31, CYR32, and CYR33.

Agronomic performance of R2207. Line R2207 showed neither morphological nor cytological segregation after strict bagging for self-pollinating for three consecutive generations. By comparison with six representative commercial cultivars in the production, R2207 had significantly higher spike number per plant, spike length, and fertile spikelet number per spike, as well as comparable or superior kernel number per spike, thousand-kernel weight, and grain yield per plant, suggesting that R2207 could be a valuable resource for wheat improvement (Fig. 6, Table 3).

Use of R2207 in wheat breeding. To efficiently transfer the resistance gene(s) into different wheat backgrounds, R2207 was crossed with 12 commercial cultivars including Shixin633, Shixin733, Shixin828, Gao8901, Shiyou17, Shixin5071, Lumai13, Liangxing99, and so on. The $\mathrm{F}_{2}$ or $\mathrm{BC}_{1} \mathrm{~F}_{1}$ progenies of those crosses were selected using the 1RS-specific KASP marker SWK5282 (Fig. 7). The powdery mildew resistance of the offspring plants cosegregated with the detection results of the $1 \mathrm{RS}$ alleles and was not restricted to the different wheat backgrounds. Based on the genotyping results, combined with the phenotype evaluation, the highly resistant derivative plants against powdery mildew and stripe rust with the 1RS alleles and desirable agronomic performance have been retained for further MAS breeding.

\section{Discussion}

Rye, a naturally cross-pollinating relative of common wheat, is an important gene donor for wheat improvement. Through distant

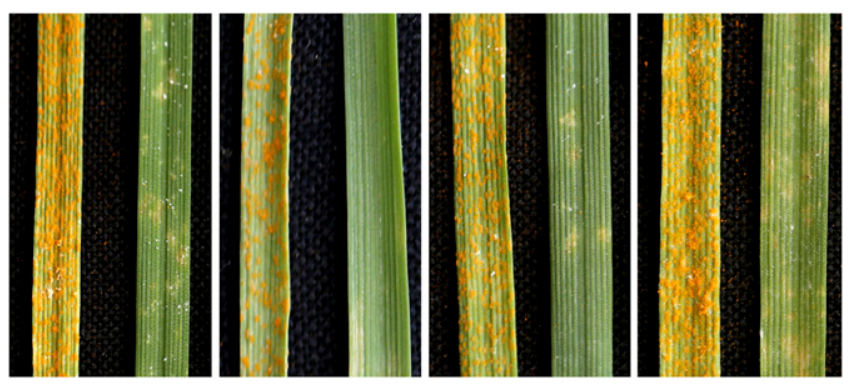

Fig. 5. Stripe rust response of Mingxian169 and R2207 to races CYR31, CYR32, CYR33, and CYR34 of Puccinia striiformis f. sp. tritici at the seedling stage, respectively.

Table 2. Seedling disease responses of R2207 and susceptible wheat cultivar Mingxian169 to races CYR31, CYR32, CYR33, and CYR34 of Puccinia striiformis f. sp. tritici

\begin{tabular}{|c|c|c|c|}
\hline \multirow[b]{2}{*}{ Races } & \multirow[b]{2}{*}{ Virulence spectra on $Y r$ genes $^{y}$} & \multicolumn{2}{|c|}{ Infection types $^{z}$} \\
\hline & & R2207 & Mingxian169 \\
\hline CYR31 & $\begin{array}{l}\operatorname{Yr} 1, \operatorname{Yr} 6, \operatorname{Yr} 7, \operatorname{Yr} 8, \operatorname{Yr} 9, \operatorname{Yr} 17, \operatorname{Yr} 18, \operatorname{Yr} 28, \\
\quad \operatorname{Yr} 29, \operatorname{Yr} 43, \operatorname{Yr} 44, \operatorname{YrExp} 2, \operatorname{YrSP}\end{array}$ & 0 & 4 \\
\hline CYR32 & $\begin{array}{l}Y r 1, \operatorname{Yr} 6, \operatorname{Yr} 7, \operatorname{Yr} 8, \operatorname{Yr} 9, \operatorname{Yr} 17, \operatorname{Yr} 18, \operatorname{Yr} 27, \\
\quad \operatorname{Yr} 28, \operatorname{Yr} 29, \operatorname{Yr} 31, \operatorname{Yr} 43, \operatorname{Yr} 44, \operatorname{YrExp} 2 \\
\operatorname{YrSP}\end{array}$ & 0 & 4 \\
\hline CYR33 & $\begin{array}{l}Y r 1, Y r 6, Y r 7, Y r 9, Y r 17, \operatorname{Yr} 18, Y r 28, Y r 29, \\
\text { Yr31, Yr43, Yr44, YrExp2, YrSP }\end{array}$ & 0 & 4 \\
\hline CYR34 & $\begin{array}{l}Y r 1, Y r 6, Y r 7, \operatorname{Yr} 8, \operatorname{Yr} 9, \operatorname{Yr} 10, \operatorname{Yr} 17, \operatorname{Yr} 18, \\
\quad \text { Yr24, Yr26, Yr27, Yr28, Yr29, Yr31, } \\
\text { Yr32, Yr43, Yr44, YrExp2, YrSP }\end{array}$ & 0 & 4 \\
\hline
\end{tabular}

hybridization and chromosome engineering, many wheat-rye addition, substitution, and translocation lines have been developed for further use in wheat breeding. Compared with addition lines and substitution lines, translocation lines are preferred by breeders because of less linkage drag, smaller alien genetic segments, and regular meiotic behavior (Falke et al. 2009). In the present study, a novel wheat-rye T1RS·1BL translocation line (R2207) was systematically characterized by GISH, mc-FISH with multiple probes, mc-GISH, molecular marker analysis, multiple pathogen tests, and agronomic traits assessment. Line R2207 possessed broad resistance to powdery mildew that was most probably conferred by a new variation on chromosome 1RS. Also, it has superior resistance to stripe rust at both seedling and adult-plant stages and enhanced agronomic traits; thus, it should be a promising disease resistance donor for wheat improvement. Among all of the rye translocation lines, 1RS translocation line was regarded as a particularly notable success in wheat breeding (Tester and Langridge 2010). Since the 1950s, when the 1RS chromosome arm from the German rye cultivar Petkus was first transferred into common wheat (Schlegel and Korzun 1997), the wheat-rye T1RS·1BL translocations have made great contributions to wheat improvement due to their multiple disease resistance and superior yield traits. Unfortunately, owing to continual evolution of virulent isolates in the pathogen populations, the resistance genes on 1RS have successively lost their resistance. After large numbers of released cultivars carrying T1RS·1BL translocation lost their disease resistance, the problem of a single source of origin causing a lack of genetic diversity was extensively revealed. Researchers have made great efforts to create new T1RS $1 \mathrm{BL}$ translocation lines from different resources (Li et al. 2016; Lukaszewski 2017; Molnár-Láng

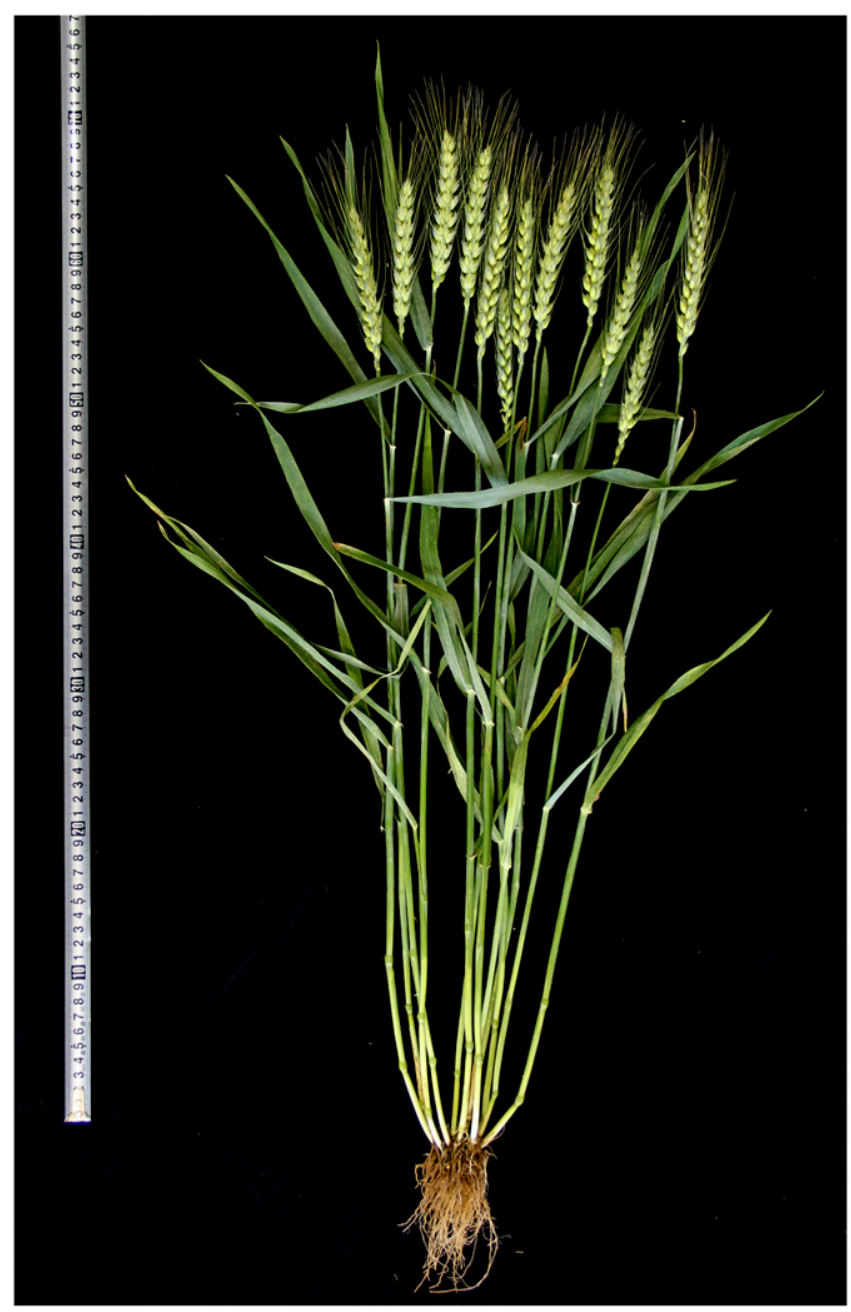

Fig. 6. Morphological traits of R2207 plant. 
et al. 2010; Qi et al. 2016; Ren et al. 2009, 2017, 2018; Yang et al. 2014). Those new T1RS·1BL translocation lines had either valuable resistance traits, or elite agronomic performance, or other elite genes/ alleles, and hence served as new valuable donors for wheat improvement. Therefore, there is still potential to identify more new elite variations to broaden the genetic base, to enhance the disease resistance, and simultaneously to improve the yield traits. For this reason, R2207 is undoubtedly a promising resource for disease resistance breeding.

Rye is a naturally cross-pollinating crop, resulting in heterogeneity between and within different cultivars. This characteristic creates huge variation in rye for wheat improvement, which, to some extent, explained why new 1 RS variations have been continuously identified. Many instances have demonstrated this phenomenon. For example, Ren et al. $(2017,2018)$ developed T1RS·1BL translocation lines derived from the rye cultivars Weining and Baili, respectively. Both of them were resistant to powdery mildew and stripe rust. Li et al. (2016) developed three new T1RS·1BL translocation lines and a new $1 \mathrm{R}$ (1B) substitution line. Surprisingly although the four lines were all derived from the rye cultivar Aigan and the susceptible wheat cultivar Mianyang11, their responses to inoculated Pst pathotypes turned out to be significantly different from each other. This may due to the high genetic diversity in rye. There exists a high probability of genetic diversity in rye cultivars (Ren et al. 2011). In the present study, R2207 showed significantly different reaction patterns to $23 \mathrm{Bgt}$ isolates from those of $\mathrm{Pm} 7, \mathrm{Pm} 8, \mathrm{Pm} 17$, and $\mathrm{Pm} 20$ (Table 1). Combined with different reaction patterns of R2207 to pathogen isolates, the cosegregation of resistance with $1 \mathrm{RS}$ in $\mathrm{F}_{2}$ individuals indicated that it should possess a new resistance variation for powdery mildew on 1RS. Meanwhile, R2207 exhibited superior resistance to four $P$ st races, especially against the widely virulent race CYR34 (Fig. 5, Table 2), which further revealed its high breeding value in wheat production. Of course, more genetic and molecular evidence between R2207 and other reported resistance genes on $1 \mathrm{RS}$ is still required to further determine their differences. Fine genomic sequencing of different wheat cultivars and the cloning of the key genes and their functional validation should explain the evolutionary relationship between the documented resistance stocks.

Although many 1RS translocation lines have been developed, not every line is useful for breeding (Carver and Rayburn 1995; Kim et al. 2004; McKendry et al. 1996; Singh et al. 1998; Villareal et al. 1998; Zhou et al. 2007). Several reports indicated that T1RS $1 \mathrm{BL}$ translocations increased yield in only specific combinations of 1RS and wheat genetic background (Jung and Lelley 1985; Ren et al. 2017). Fortunately, R2207 showed elite agronomic performance accompanied with resistance to two diseases. Compared with three commercial cultivars carrying T1RS·1BL translocations (Shimai18, Ji5265, and Heng4444) and three commercial cultivars without T1RS·1BL translocations (Shixin633, Shixin733, and Shixin828), R2207 showed significant enhancement of spike number per plant, spike length, and fertile spikelet number per spike, and comparable or superior levels of kernel number per spike, thousand-kernel weight, and grain yield per plant. Meanwhile, R2207 exhibited high resistance at adult stage to the mixed $B g t$ isolates in the field and to 16 of $23 \mathrm{Bgt}$ isolates tested at the seedling stage, and it also showed high resistance to the mixture of Pst races at the adult stage and Pst races CYR31, CYR32, CYR33, and CYR34 at the seedling stage. Therefore, R2207combined multiple valuable traits and could be a promising donor for wheat breeding.

To transfer the T1RS·1BL translocation into different wheat genetic backgrounds, 1RS-specific KASP marker SWK5282 was used to efficiently select the progenies of R2207 crossing with cultivars such as Shixin633, Shixin733, Shixin828, Gao8901, and so on. In this process, SWK5282 was shown to be more time-saving and convenient than traditional PCR markers and greatly accelerated the transfer of resistance genes and achieved high-throughput detection (Han et al. 2020). Then, combined with the phenotype investigation in the field, $\mathrm{F}_{2}$ or $\mathrm{BC}_{1} \mathrm{~F}_{1}$ individuals with the $1 \mathrm{RS}$ allele and elite agronomic traits were selected, and the seeds were sown in the field. In the near future, more stable high-generation lines derived from R2207 should be obtained and serve for wheat improvement.

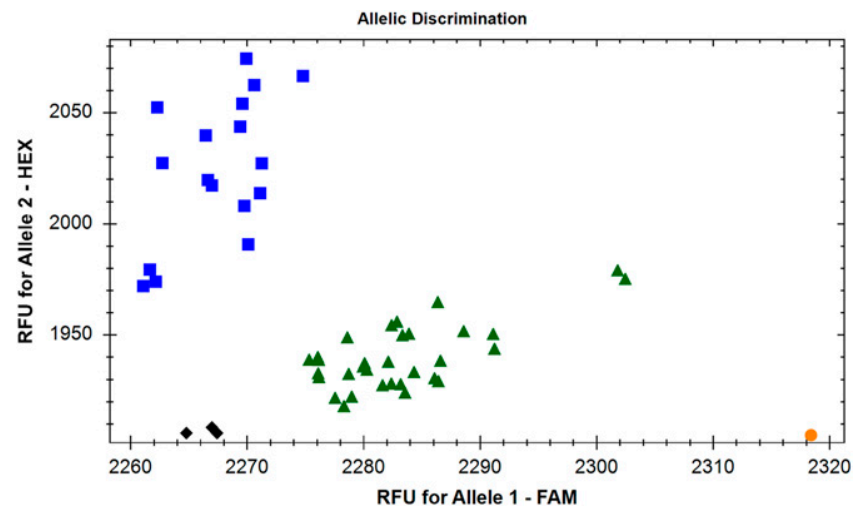

Fig. 7. The genotyping results of the 1 RS-specific kompetitive allele specific PCR marker performed for marker-assisted selection in $F_{2}$ progenies of R2207 and commercial cultivar Shixin633. Orange circles represent the homozygous 1RS special single nucleotide polymorphism (SNP) (Allele 1/Allele 1), indicating control rye Imperial; blue squares represent the homozygous wheat special SNP (Allele 2/ Allele 2), indicating wheat parent Shixin633, the $F_{2}$ plants without chromosome arms 1RS, and control Chinese Spring. Green triangles represent the presence of both Allele 1 and Allele 2, indicating R2207 and the $F_{2}$ plants containing chromosome arms $1 \mathrm{RS}$. Black diamonds indicate no template control.

Table 3. Agronomic performance of R2207 compared with six representative commercial cultivars ${ }^{\mathrm{z}}$

\begin{tabular}{|c|c|c|c|c|c|c|c|c|}
\hline Year & Lines & $\begin{array}{c}\text { Plant height } \\
(\mathbf{c m})\end{array}$ & $\begin{array}{c}\text { Spike number } \\
\text { per plant }\end{array}$ & $\begin{array}{c}\text { Spike } \\
\text { length }(\mathbf{c m})\end{array}$ & $\begin{array}{c}\text { Fertile spikelet } \\
\text { number per spike }\end{array}$ & $\begin{array}{c}\text { Kernel number } \\
\text { per spike }\end{array}$ & $\begin{array}{l}\text { Thousand-kernel } \\
\text { weight (g) }\end{array}$ & $\begin{array}{c}\text { Grain yield per } \\
\text { plant (g) }\end{array}$ \\
\hline \multirow[t]{7}{*}{ 2015-2016 } & R2207 & $76.0 \pm 1.4 \mathrm{a}$ & $12.7 \pm 1.3 \mathrm{a}$ & $11.8 \pm 0.2 \mathrm{a}$ & $22.3 \pm 1.3 \mathrm{a}$ & $72.0 \pm 2.9 \mathrm{a}$ & $48.3 \pm 1.1 \mathrm{ab}$ & $33.4 \pm 2.7 \mathrm{a}$ \\
\hline & Shimai18 & $68.3 \pm 2.7 \mathrm{c}$ & $10.5 \pm 0.9 \mathrm{c}$ & $10.0 \pm 0.3 \mathrm{c}$ & $18.7 \pm 1.2 \mathrm{c}$ & $61.1 \pm 1.6 \mathrm{~d}$ & $48.0 \pm 1.7 \mathrm{ab}$ & $26.3 \pm 2.4 \mathrm{c}$ \\
\hline & Ji5265 & $66.2 \pm 1.5 \mathrm{~d}$ & $11.5 \pm 1.2 \mathrm{~b}$ & $8.9 \pm 0.5 \mathrm{~d}$ & $19.8 \pm 0.6 b$ & $67.5 \pm 3.1 \mathrm{~b}$ & $45.8 \pm 1.4 \mathrm{c}$ & $30.5 \pm 3.2 \mathrm{ab}$ \\
\hline & Heng4444 & $71.2 \pm 1.8 \mathrm{~b}$ & $9.5 \pm 1.1 \mathrm{~d}$ & $10.6 \pm 0.4 \mathrm{~b}$ & $17.4 \pm 1.2 \mathrm{~d}$ & $64.1 \pm 3.2 \mathrm{c}$ & $49.2 \pm 1.5 \mathrm{ab}$ & $27.0 \pm 3.4 b c$ \\
\hline & Shixin633 & $65.7 \pm 2.2 \mathrm{~d}$ & $9.2 \pm 1.0 \mathrm{~d}$ & $9.1 \pm 0.4 \mathrm{~d}$ & $19.0 \pm 1.1 b c$ & $64.3 \pm 3.3 \mathrm{c}$ & $47.4 \pm 1.4 b c$ & $26.1 \pm 4.0 \mathrm{c}$ \\
\hline & Shixin733 & $70.5 \pm 1.5 \mathrm{~b}$ & $7.3 \pm 1.1 \mathrm{e}$ & $8.3 \pm 0.2 \mathrm{e}$ & $18.5 \pm 0.5 \mathrm{c}$ & $70.6 \pm 2.8 \mathrm{a}$ & $49.8 \pm 1.6 \mathrm{a}$ & $24.1 \pm 2.2 \mathrm{c}$ \\
\hline & Shixin828 & $68.8 \pm 1.2 \mathrm{c}$ & $8.0 \pm 1.0 \mathrm{e}$ & $8.9 \pm 0.3 \mathrm{~d}$ & $17.5 \pm 0.5 \mathrm{~d}$ & $70.9 \pm 2.5 \mathrm{a}$ & $47.9 \pm 1.4 \mathrm{ab}$ & $24.0 \pm 4.2 \mathrm{c}$ \\
\hline \multirow[t]{7}{*}{ 2016-2017 } & R2207 & $74.2 \pm 2.1 \mathrm{a}$ & $13.9 \pm 1.2 \mathrm{a}$ & $11.6 \pm 0.6 \mathrm{a}$ & $20.5 \pm 0.7 \mathrm{a}$ & $62.5 \pm 2.2 \mathrm{a}$ & $43.7 \pm 1.4 \mathrm{a}$ & $31.1 \pm 3.8 \mathrm{a}$ \\
\hline & Shimai18 & $63.7 \pm 1.6 \mathrm{e}$ & $12.0 \pm 1.3 \mathrm{bc}$ & $9.1 \pm 0.2 \mathrm{~b}$ & $17.0 \pm 0.6 b c$ & $54.1 \pm 3.2 \mathrm{c}$ & $43.1 \pm 1.6 \mathrm{ab}$ & $25.0 \pm 2.7 b c$ \\
\hline & Ji5265 & $64.3 \pm 1.3 \mathrm{de}$ & $12.6 \pm 1.3 b$ & $8.3 \pm 0.3 c$ & $17.5 \pm 0.8 b$ & $61.8 \pm 2.3 \mathrm{a}$ & $40.7 \pm 1.6 \mathrm{c}$ & $27.2 \pm 1.9 \mathrm{~b}$ \\
\hline & Heng4444 & $69.4 \pm 1.9 \mathrm{~b}$ & $11.5 \pm 1.2 \mathrm{c}$ & $8.9 \pm 0.2 b$ & $16.1 \pm 0.7 \mathrm{~d}$ & $57.8 \pm 2.4 \mathrm{~b}$ & $44.0 \pm 1.6 \mathrm{a}$ & $26.3 \pm 2.9 \mathrm{~b}$ \\
\hline & Shixin633 & $65.2 \pm 1.3 \mathrm{de}$ & $11.2 \pm 1.2 \mathrm{~cd}$ & $8.4 \pm 0.3 c$ & $17.2 \pm 0.6 \mathrm{~b}$ & $58.1 \pm 3.2 \mathrm{~b}$ & $41.0 \pm 2.0 \mathrm{bc}$ & $24.4 \pm 3.2 \mathrm{bc}$ \\
\hline & Shixin733 & $67.5 \pm 2.2 \mathrm{c}$ & $10.0 \pm 1.3 \mathrm{e}$ & $8.2 \pm 0.2 c$ & $17.0 \pm 0.9 \mathrm{bc}$ & $60.9 \pm 1.7 \mathrm{a}$ & $44.7 \pm 1.9 \mathrm{a}$ & $23.9 \pm 2.3 b c$ \\
\hline & Shixin828 & $65.4 \pm 1.8 \mathrm{~d}$ & $10.4 \pm 1.3 \mathrm{de}$ & $8.3 \pm 0.3 c$ & $16.4 \pm 1.0 \mathrm{~cd}$ & $61.5 \pm 1.8 \mathrm{a}$ & $42.9 \pm 1.5 \mathrm{ab}$ & $22.6 \pm 2.6 \mathrm{c}$ \\
\hline
\end{tabular}

\footnotetext{
${ }^{\mathrm{z}}$ Values followed by the same letters in the same column were not significantly different at the 0.05 probability level according to the LSD test.
} 
In conclusion, this study provides a thorough methodology for identifying a novel wheat-rye T1RS·1BL translocation line with superior agronomic traits and high resistance to two diseases as well as an effective method for transferring the chromosome 1RS for MAS. In the future, the development of more T1RS $1 \mathrm{BL}$ translocation lines should be encouraged to increase genetic diversity and fully release the potential of $1 \mathrm{RS}$ for wheat breeding. Meanwhile, small segment translocation lines of R2207 will be produced for accurate utilization of the valuable traits in $1 \mathrm{RS}$ in the near future.

\section{Acknowledgments}

The authors are grateful to Prof. Shichang Xu (Institute of Plant Protection, Chinese Academy of Agricultural Sciences, Beijing) for kindly providing stripe rust races and to Dr. John Raupp (Kansas State University, Manhattan, KS) for the rye cultivar Imperial, the complete set of disomic addition lines of CS-Imperial, and $1 \mathrm{RS}$ and 1RL ditelosomic addition lines of CS-Imperial.

\section{Literature Cited}

An, D. G., Li, L. H., Li, J. M., Li, H. J., and Zhu, Y. G. 2006. Introgression of resistance to powdery mildew conferred by chromosome $2 \mathrm{R}$ by crossing wheat nullisomic 2D with rye. J. Integr. Plant Biol. 48:838-847.

An, D. G., Ma, P. T., Zheng, Q., Fu, S. L., Li, L. H., Han, F. P., Han, G. H., Wang, J., Xu, Y. F., Jin, Y. L., Luo, Q. L., and Zhang, X. T. 2019. Development and molecular cytogenetic identification of a new wheat-rye 4R chromosome disomic addition line with resistances to powdery mildew, stripe rust and sharp eyespot. Theor. Appl. Genet. 132:257-272.

An, D. G., Zheng, Q., Zhou, Y. L., Ma, P. T., Lv, Z. L., Li, L. H., Li, B., Luo, Q. L., $\mathrm{Xu}, \mathrm{H}$. X., and Xu, Y. F. 2013. Molecular cytogenetic characterization of a new wheat-rye $4 \mathrm{R}$ chromosome translocation line resistant to powdery mildew. Chromosome Res. 21:419-432.

Carver, B. F., and Rayburn, A. L. 1995. Comparison of related wheat stocks possessing 1B or T1BL.1RS chromosomes: Grain and flour quality. Crop Sci. 35:1316-1321.

Chen, W. Q., Wellings, C., Chen, X. M., Kang, Z. S., and Liu, T. G. 2014. Wheat stripe (yellow) rust caused by Puccinia striiformis f. sp. tritici. Mol. Plant Pathol. 15:433-446.

Chen, W. Q., Wu, L. R., Liu, T. G., Xu, S. C., Jin, S. L., Peng, Y. L., and Wang, B. T. 2009. Race dynamics, diversity, and virulence evolution in Puccinia striiformis f. sp. tritici, the causal agent of wheat stripe rust in China from 2003 to 2007. Plant Dis. 93:1093-1101.

Chen, X. M. 2013. Review article: high-temperature adult-plant resistance, key for sustainable control of stripe rust. Am. J. Plant Sci. 4:608-627.

Chen, X. M. 2014. Integration of cultivar resistance and fungicide application for control of wheat stripe rust. Can. J. Plant Pathol. 36:311-326.

Cowger, C., Mehra, L., Arellano, C., Meyers, E., and Murphy, J. P. 2018. Virulence differences in Blumeria graminis f. sp. tritici from the central and eastern United States. Phytopathology 108:402-411.

Cowger, C., Parks, R., and Marshall, D. 2009. Appearance of powdery mildew of wheat caused by Blumeria graminis f. sp. tritici on Pm17-bearing cultivars in North Carolina. Plant Dis. 93:1219.

Dean, R., Kan, J. A. L. V., Pretorius, Z. A., Hammond-Kosack, K. E., Pietro, A. D., Spanu, P. D., Rudd, J. J., Dickman, M., Kahmann, R., Ellis, J., and Foster, G. D. 2012. The top 10 fungal pathogens in molecular plant pathology. Mol. Plant Pathol. 13:414-430.

Dvorak, J., Wang, L., Zhu, T. T., Jorgensen, C. M., Luo, M. C., Deal, K. R., Gu, Y. Q., Gill, B. S., Distelfeld, A., Devos, K. M., Qi, P., and McGuire, P. E. 2018. Reassessment of the evolution of wheat chromosomes $4 \mathrm{~A}, 5 \mathrm{~A}$, and $7 \mathrm{~B}$. Theor. Appl. Genet. 131:2451-2462.

Edet, O. U., Kim, J. S., Okamoto, M., Hanada, K., Takeda, T., Kishii, M., Gorafi, Y. S. A., and Tsujimoto, H. 2018. Efficient anchoring of alien chromosome segments introgressed into bread wheat by new Leymus racemosus genomebased markers. BMC Genet. 19:18.

Falke, K. C., Sušić, Z., Wilde, P., Wortmann, H., Möhring, J., Piepho, H. P., Geiger, H. H., and Miedaner, T. 2009. Testcross performance of rye introgression lines developed by marker-assisted backcrossing using an Iranian accession as donor. Theor. Appl. Genet. 118:1225-1238.

Fu, S. L., Lv, Z. L., Qi, B., Guo, X., Li, J., Liu, B., and Han, F. P. 2012. Molecular cytogenetic characterization of wheat-Thinopyrum elongatum addition, substitution and translocation lines with a novel source of resistance to wheat Fusarium head blight. J. Genet. Genomics 39:103-110.

Gebreslasie, Z. S., Huang, S., Zhan, G. M., Badebo, A., Zeng, Q. D., Wu, J. H., Wang, Q. L., Liu, S. J., Huang, L. L., Wang, X. J., Kang, Z. S., and Han, D. J. 2020. Stripe rust resistance genes in a set of Ethiopian bread wheat cultivars and breeding lines. Euphytica 216:17.

Gill, K. S., Lubbers, E. L., Gill, B. S., Raupp, W. J., and Cox, T. S. 1991. A genetic linkage map of Triticum tauschii (DD) and its relationship to the D genome of bread wheat (AABBDD). Genome 34:362-374.

Gupta, P. K., Langridge, P., and Mir, R. R. 2010. Marker-assisted wheat breeding: present status and future possibilities. Mol. Breed. 26:145-161.
Han, D. J., Wang, Q. L., Chen, X. M., Zeng, Q. D., Wu, J. H., Xue, W. B., Zhan, G. M., Huang, L. L., and Kang, Z. S. 2015. Emerging Yr26-virulent races of Puccinia striiformis f. sp. tritici are threatening wheat production in the Sichuan Basin, China. Plant Dis. 99:754-760.

Han, F. P., Gao, Z., and Birchler, J. A. 2009. Reactivation of an inactive centromere reveals epigenetic and structural components for centromere specification in maize. Plant Cell 21:1929-1939.

Han, G. H., Liu, S. Y., Jin, Y. L., Jia, M. S., Ma, P. T., Liu, H., Wang, J., and An, D. G. 2020. Scale development and utilization of universal PCR-based and high-throughput KASP markers specific for chromosome arms of rye (Secale cereale L.). BMC Genomics 21:206.

Hechanova, S. L., Prusty, M. R., Kim, S. R., Ballesfin, L., Ramos, J., Prahalada, G. D., and Jena, K. K. 2018. Monosomic alien addition lines (MAALs) of Oryza rhizomatis in Oryza sativa: Production, cytology, alien trait introgression, molecular analysis and breeding application. Theor. Appl. Genet. 131:2197-2211

Howell, T., Hale, I., Jankuloski, L., Bonafede, M., Gilbert, M., and Dubcovsky, J. 2014. Mapping a region within the 1 RS.1BL translocation in common whea affecting grain yield and canopy water status. Theor. Appl. Genet. 127: 2695-2709.

Howell, T., Moriconi, J. I., Zhao, X. Q., Hegarty, J., Fahima, T., Santa-Maria, G. E., and Dubcovsky, J. 2019. A wheat/rye polymorphism affects seminal root length and yield across different irrigation regimes. J. Exp. Bot. 70: 4027-4037.

Jung, C., and Lelley, T. 1985. Genetic interaction between wheat and rye genomes in triticale. Theor. Appl. Genet. 70:427-432.

Kim, W., Johnson, J. W., Baenziger, P. S., Lukaszewski, A. J., and Gaines, C. S. 2004. Agronomic effect of wheat-rye translocation carrying rye chromatin (1R) from different sources. Crop Sci. 44:1254-1258.

Kumlay, A. M., Baenziger, P. S., Gill, K. S., Shelton, D. R., Graybosch, R. A. Lukaszewski, A. J., and Wesenberg, D. M. 2003. Understanding the effect of rye chromatin in bread wheat. Crop Sci. 43:1643-1651.

Li, H. H., Dong, Z. J., Ma, C., Xia, Q., Tian, X. B., Sehgal, S., Koo, D. H., Friebe, B., Ma, P. T., and Liu, W. X. 2020b. A spontaneous wheat-Aegilops longissima translocation carrying Pm66 confers resistance to powdery mildew. Theor Appl. Genet. 133:1149-1159.

Li, H. J., Wang, X. M., Song, F. J., Wu, C. P., Wu, X. F., Zhang, N., Zhou, Y., and Zhang, X. Y. 2011. Response to powdery mildew and detection of resistance genes in wheat cultivars from China. Acta Agron. Sin. 37:943-954.

Li, J. B., Dundas, I., Dong, C. M., Li, G. R., Trethowan, R., Yang, Z. J., Hoxha, S., and Zhang, P. 2020a. Identification and characterization of a new stripe rust resistance gene $\operatorname{Yr} 83$ on rye chromosome 6R in wheat. Theor. Appl. Genet. 133:1095-1107.

Li, Z., Ren, Z. L., Tan, F. Q., Tang, Z. X., Fu, S. L., Yan, B. J., and Ren, T. H. 2016. Molecular cytogenetic characterization of new wheat-rye $1 \mathrm{R}(1 \mathrm{~B})$ substitution and translocation lines from a Chinese Secale cereal L. Aigan with resistance to stripe rust. PLoS One 11:e016364.

Lukaszewski, A. J. 1990. Frequency of 1RS.1AL and 1RS.1BL translocations in United States wheats. Crop Sci. 30:1151-1153.

Lukaszewski, A. J. 2017. A set of new 1RS translocations from wheat cv. Amigo in a uniform genetic background. Euphytica 213:214.

Lutz, J., Limpert, E., Bartoš, P., and Zeller, F. J. 1992. Identification of powdery mildew resistance genes in common wheat (Triticum aestivum L.). I Czechoslovakian cultivars. Plant Breed. 108:33-39.

Ma, P. T., Han, G. H., Zheng, Q., Liu, S. Y., Han, F. P., Wang, J., Luo, Q. L., and An, D. G. 2020. Development of novel wheat-rye chromosome 4R translocations and assignment of their powdery mildew resistance. Plant Dis. 104:260-268

Mago, R., Miah, H., Lawrence, G. J., Wellings, C. R., Spielmeyer, W., Bariana, H. S., McIntosh, R. A., Pryor, A. J., and Ellis, J. G. 2005. High-resolution mapping and mutation analysis separate the rust resistance genes $\operatorname{Sr} 31, \operatorname{Lr} 26$ and $Y r 9$ on the short arm of rye chromosome 1. Theor. Appl. Genet. 112:41-50.

Mago, R., Zhang, P., Vautrin, S., Šimková, H., Bansal, U., Luo, M. C., Rouse, M., Karaoglu, H., Periyannan, S., Kolmer, J., Jin, Y., Ayliffe, M. A., Bariana, H., Park, R. F., McIntosh, R., Doležel, J., Bergès, H., Spielmeyer, W., Lagudah, E. S., Ellis, J. G., and Dodds, P. N. 2015. The wheat Sr50 gene reveals rich diversity at a cereal disease resistance locus. Nat. Plants 1:15186.

McIntosh, R. A., Dubcovsky, J., Rogers, W. J., Xia, X. C., and Raupp, W. J. 2019 Catalogue of gene symbols for wheat: 2019 supplement. Pages 98-113 in: Annual Wheat Newsletter. W. J. Raupp, ed. Wheat Genetic and Genomic Resources at Kansas State University, Manhattan, KS.

McIntosh, R. A., Zhang, P., Cowger, C., Parks, R., Lagudah, E. S., and Hoxha, S. 2011. Rye-derived powdery mildew resistance gene $P m 8$ in wheat is suppressed by the Pm3 locus. Theor. Appl. Genet. 123:359-367.

McKendry, A. L., Tague, D. N., and Miskin, K. E. 1996. Effect of 1BL.1RS on agronomic performance of soft red winter wheat. Crop Sci. 36:844-847.

Molnár-Láng, M., Cseh, A., Szakács, É., and Molnár, I. 2010. Development of a wheat genotype combining the recessive crossability alleles $k r 1 k r 1 k r 2 k r 2$ and the 1BL.1RS translocation, for the rapid enrichment of 1RS with new allelic variation. Theor. Appl. Genet. 120:1535-1545.

Mujeeb-Kazi, A., Kazi, A. G., Dundas, I., Rasheed, A., Ogbonnaya, F., Kishii, M., Bonnett, D., Wang, R. R. C., Xu, S. S., Chen, P. D., Mahmood, T., Bux, H., and 
Farrakh, S. 2013. Genetic diversity for wheat improvement as a conduit for food security. Adv. Agron. 122:179-257.

Parks, R., Carbone, I., Murphy, J. P., Marshall, D., and Cowger, C. 2008. Virulence structure of the eastern U.S. wheat powdery mildew population. Plant Dis. 92:1074-1082.

Pretorius, Z. A., Bender, C. M., Visser, B., and Terefe, T. 2010. First report of a Puccinia graminis f. sp. tritici race virulent to the $S r 24$ and $S r 31$ wheat stem rust resistance genes in South Africa. Plant Dis. 94:784.

Pretorius, Z. A., Singh, R. P., Wagoire, W. W., and Payne, T. S. 2000. Detection of virulence to wheat stem rust resistance gene Sr31 in Puccinia graminis f. sp. tritici in Uganda. Plant Dis. 84:203.

Qi, W. L., Tang, Y., Zhu, W., Li, D. Y., Diao, C. D., Xu, L. L., Zeng, J., Wang, Y., Fan, X., Sha, L. N., Zhang, H. Q., Zheng, Y. L., Zhou, Y. H., and Kang, H. Y. 2016. Molecular cytogenetic characterization of a new wheat-rye $1 \mathrm{BL} \cdot 1 \mathrm{RS}$ translocation line expressing superior stripe rust resistance and enhanced grain yield. Planta 244:405-416.

Qie, Y. M., Sheng, Y., Xu, H. X., Jin, Y. L., Ma, F. F., Li, L. H., Li, X. Q., and An, D. G. 2019. Identification of a new powdery mildew resistance gene $p m D H T$ at or closely linked to the Pm5 locus in the Chinese wheat landrace Dahongtou. Plant Dis. 103:2645-2651.

Rabinovich, S. V. 1998. Importance of wheat-rye translocations for breeding modern cultivars of Triticum aestivum L. [Reprinted from Wheat: Prospects for Global Improvement]. Euphytica 100:323-340.

Rahmatov, M., Rouse, M. N., Nirmala, J., Danilova, T., Friebe, B., Steffenson, B. J., and Johansson, E. 2016. A new 2DS·2RL Robertsonian translocation transfers stem rust resistance gene Sr59 into wheat. Theor. Appl. Genet. 129:1383-1392.

Rasheed, A., Wen, W., Gao, F. M., Zhai, S. N., Jin, H., Liu, J. D., Guo, Q., Zhang, Y. J., Dreisigacker, S., Xia, X. C., and He, Z. H. 2016. Development and validation of KASP assays for genes underpinning key economic traits in bread wheat. Theor. Appl. Genet. 129:1843-1860.

Ren, T. H., Chen, F., Zou, Y. T., Jia, Y. H., Zhang, H. Q., Yan, B. J., and Ren, Z. L. 2011. Evolutionary trends of microsatellites during the speciation process and phylogenetic relationships within the genus Secale. Genome 54:316-326.

Ren, T. H., Ren, Z. L., Yang, M. Y., Yan, B. J., Tan, F. Q., Fu, S. L., Tang, Z. X., and Li, Z. 2018. Novel source of $1 \mathrm{RS}$ from Baili rye conferred high resistance to diseases and enhanced yield traits to common wheat. Mol. Breed. 38:101.

Ren, T. H., Tang, Z. X., Fu, S. L., Yan, B. J., Tan, F. Q., Ren, Z. L., and Li, Z. 2017. Molecular cytogenetic characterization of novel wheat-rye T1RS.1BL translocation lines with high resistance to diseases and great agronomic traits. Front. Plant Sci. 8:799.

Ren, T. H., Yang, Z. J., Yan, B. J., Zhang, H. Q., Fu, S. L., and Ren, Z. L. 2009. Development and characterization of a new 1BL.1RS translocation line with resistance to stripe rust and powdery mildew of wheat. Euphytica 169:207-213.

Schlegel, R., and Korzun, V. 1997. About the origin of 1RS.1BL wheat-rye chromosome translocations from Germany. Plant Breed. 116:537-540.

Schneider, A., Rakszegi, M., Molnár-Láng, M., and Szakács, É. 2016. Production and cytomolecular identification of new wheat-perennial rye (Secale cereanum) disomic addition lines with yellow rust resistance (6R) and increased arabinoxylan and protein content (1R, 4R, 6R). Theor. Appl. Genet. 129:1045-1059.

Semagn, K., Babu, R., Hearne, S., and Olsen, M. 2014. Single nucleotide polymorphism genotyping using kompetitive allele specific PCR (KASP): Overview of the technology and its application in crop improvement. Mol. Breed. 33:1-14.

Shearman, V. J., Sylvester-Bradley, R., Scott, R. K., and Foulkes, M. J. 2005. Physiological processes associated with wheat yield progress in the UK. Crop Sci. 45:175-185

Sheng, B. Q., and Duan, X. Y. 1991. Improvement of scale 0-9 method for scoring adult plant resistance to powdery mildew of wheat. Beijing Agr. Sci. 1:38-39.

Shimizu, Y., Nasuda, S., and Endo, T. R. 1997. Detection of Sec-1 locus of rye by a PCR-based method. Genes Genet. Syst. 72:197-203.
Si, Q. M., Zhang, X. X., Duan, X. Y., Sheng, B. Q., and Zhou, Y. L. 1992. On gene analysis and classification of powdery mildew (Erysiphe graminis f. sp. tritici) resistant wheat varieties. Acta Phytopathol. Sin. 22:349-355.

Singh, R. P., Huerta-Espino, J., Rajaram, S., and Crossa, J. 1998. Agronomic effects from chromosome translocations 7DL.7Ag and 1BL.1RS in spring wheat. Crop Sci. 38:27-33.

Tan, C. C., Li, G. Q., Cowger, C., Carver, B. F., and Xu, X. Y. 2018. Characterization of Pm59, a novel powdery mildew resistance gene in Afghanistan wheat landrace PI 181356. Theor. Appl. Genet. 131:1145-1152.

Tang, Z. X., Yang, Z. J., and Fu, S. L. 2014. Oligonucleotides replacing the roles of repetitive sequences pAs1, pSc119.2, pTa-535, pTa71, CCS1, and pAWRC.1 for FISH analysis. J. Appl. Genet. 55:313-318.

Targońska, M., Bolibok-Brągoszewska, H., and Rakoczy-Trojanowska, M. 2016. Assessment of genetic diversity in Secale cereale based on SSR markers. Plant Mol. Biol. Report. 34:37-51.

Tester, M., and Langridge, P. 2010. Breeding technologies to increase crop production in a changing world. Science 327:818-822.

Van Campenhout, S., Vander Stappen, J., Sagi, L., and Volckaert, G. 1995. Locus specific primers for LMW glutenin genes on each of the group 1 chromosomes of hexaploid wheat. Theor. Appl. Genet. 91:313-319.

Villareal, R. L., Banuelos, O., Mujeeb-Kazi, A., and Rajaram, S. 1998. Agronomic performance of chromosome 1B and T1BL.1RS near-isolines in the spring bread wheat Seri M82. Euphytica 103:195-202.

Volin, R. B., and Sharp, E. L. 1973. Physiologic specialization and pathogen aggressiveness in stripe rust. Phytopathology 63:699-703.

Wan, A. M., Zhao, Z. H., Chen, X. M., He, Z. H., Jin, S. L., Jia, Q. Z., Yao, G., Yang, J. X., Wang, B. T., Li, G. B., Bi, Y. Q., and Yuan, Z. Y. 2004. Wheat stripe rust epidemic and virulence of Puccinia striiformis f. sp. tritici in China in 2002. Plant Dis. 88:896-904.

Wang, C. M., Zheng, Q., Li, L. H., Niu, Y. C., Wang, H. B., Li, B., Zhang, X. T., Xu, Y. F., and An, D. G. 2009. Molecular cytogenetic characterization of a new T2BL-1RS wheat-rye chromosome translocation line resistant to stripe rust and powdery mildew. Plant Dis. 93:124-129.

Wang, J., Liu, Y. L., Su, H. D., Guo, X. R., and Han, F. P. 2017. Centromere structure and function analysis in the wheat-rye translocation lines. Plant J. 91:199-207.

Wu, J. H., Wang, Q. L., Kang, Z. S., Liu, S. J., Li, H. Y., Mu, J. M., Dai, M. F., Han, D. J., Zeng, Q. D., and Chen, X. M. 2017. Development and validation of KASP-SNP markers for QTL underlying resistance to stripe rust in common wheat cultivar P10057. Plant Dis. 101:2079-2087.

Wu, J. H., Wang, X. T., Chen, N., Wang, H. Y., Yu, R., Yu, S. Z., Wang, Q. L., Huang, S., Singh, R. P., Bhavani, S., Kang, Z. S., Han, D. J., and Zeng, Q. D. 2020. Association analysis identifies new loci for resistance to Chinese $\mathrm{Yr} 26$ virulent races of the stripe rust pathogen in a diverse panel of wheat germplasm. Plant Dis. 104:1751-1762.

Xu, H. X., Zhang, J., Zhang, P., Qie, Y. M., Niu, Y. C., Li, H. J., Ma, P. T., Xu, Y. F., and An, D. G. 2014. Development and validation of molecular markers closely linked to the wheat stripe rust resistance gene $\mathrm{YrC591}$ for markerassisted selection. Euphytica 198:317-323.

Yang, M. Y., Ren, T. H., Yan, B. J., Li, Z., and Ren, Z. L. 2014. Diversity resistance to Puccinia striiformis f. sp tritici in rye chromosome arm 1RS expressed in wheat. Genet. Mol. Res. 13:8783-8793.

Zadoks, J. C., Chang, T. T., and Konzak, C. F. 1974. A decimal code for the growth stages of cereals. Weed Res. 14:415-421.

Zheng, Q., Lv, Z. L., Niu, Z. X., Li, B., Li, H. W., Xu, S. S., and Han, F. P. 2014 Molecular cytogenetic characterization and stem rust resistance of five wheat- $T$. thinopyrum ponticum partial amphiploids. J. Genet. Genomics 41:591-599.

Zhou, Y., He, Z. H., Sui, X. X., Xia, X. C., Zhang, X. K., and Zhang, G. S. 2007. Genetic improvement of grain yield and associated traits in the northern China winter wheat region from 1960 to 2000. Crop Sci. 47:245-253. 